DESY 95-221

November 1995

\title{
Inclusive Charged Particle Distributions in Deep Inelastic Scattering Events at HERA
}

\author{
ZEUS Collaboration
}

\begin{abstract}
A measurement of inclusive charged particle distributions in deep inelastic ep scattering for $\gamma^{*} p$ centre-of-mass energies $75<W<175 \mathrm{GeV}$ and momentum transfer squared $10<Q^{2}<160 \mathrm{GeV}^{2}$ from the ZEUS detector at HERA is presented. The differential charged particle rates in the $\gamma^{*} p$ centre-of-mass system as a function of the scaled longitudinal momentum, $x_{F}$, and of the transverse momentum, $p_{t}^{*}$ and $\left\langle p_{t}^{* 2}\right\rangle$, as a function of $x_{F}, W$ and $Q^{2}$ are given. Separate distributions are shown for events with (LRG) and without (NRG) a rapidity gap with respect to the proton direction. The data are compared with results from experiments at lower beam energies, with the naive quark parton model and with parton models including perturbative QCD corrections. The comparison shows the importance of the higher order QCD processes. Significant differences of the inclusive charged particle rates between NRG and LRG events at the same $W$ are observed. The value of $\left\langle p_{t}^{* 2}\right\rangle$ for LRG events with a hadronic mass $M_{X}$, which excludes the forward produced baryonic system, is similar to the $\left\langle p_{t}^{* 2}\right\rangle$ value observed in fixed target experiments at $W \approx M_{X}$.
\end{abstract}




\section{The ZEUS Collaboration}

M. Derrick, D. Krakauer, S. Magill, D. Mikunas, B. Musgrave, J. Repond, R. Stanek, R.L. Talaga, H. Zhang Argonne National Laboratory, Argonne, IL, USA ${ }^{p}$

G. Bari, M. Basile, L. Bellagamba, D. Boscherini, A. Bruni, G. Bruni, P. Bruni, G. Cara Romeo, G. Castellini ${ }^{1}$, L. Cifarelli' ${ }^{2}$, F. Cindolo, A. Contin, M. Corradi, I. Gialas ${ }^{3}$, P. Giusti, G. Iacobucci, G. Laurenti, G. Levi, A. Margotti, T. Massam, R. Nania, F. Palmonari, A. Polini, G. Sartorelli,

Y. Zamora Garcia ${ }^{4}$, A. Zichichi

University and INFN Bologna, Bologna, Italy ${ }^{f}$

A. Bornheim, J. Crittenden, K. Desch, B. Diekmann ${ }^{5}$, T. Doeker, M. Eckert, L. Feld, A. Frey, M. Geerts, M. Grothe, H. Hartmann, K. Heinloth, L. Heinz, E. Hilger, H.-P. Jakob, U.F. Katz,

S. Mengel, J. Mollen ${ }^{6}$, E. Paul, M. Pfeiffer, Ch. Rembser, D. Schramm, J. Stamm, R. Wedemeyer

Physikalisches Institut der Universität Bonn, Bonn, Germany ${ }^{c}$

S. Campbell-Robson, A. Cassidy, W.N. Cottingham, N. Dyce, B. Foster, S. George, M.E. Hayes, G.P. Heath, H.F. Heath, C.J.S. Morgado, J.A. O'Mara, D. Piccioni, D.G. Roff, R.J. Tapper, R. Yoshida

H.H. Wills Physics Laboratory, University of Bristol, Bristol, U.K. ${ }^{\circ}$

R.R. Rau

Brookhaven National Laboratory, Upton, L.I., USA ${ }^{p}$

M. Arneodo ${ }^{7}$, R. Ayad, M. Capua, A. Garfagnini, L. Iannotti, M. Schioppa, G. Susinno

Calabria University, Physics Dept.and INFN, Cosenza, Italy ${ }^{f}$

A. Bernstein, A. Caldwell ${ }^{8}$, N. Cartiglia, J.A. Parsons, S. Ritz ${ }^{9}$, F. Sciulli, P.B. Straub, L. Wai, S. Yang, Q. Zhu Columbia University, Nevis Labs., Irvington on Hudson, N.Y., USA ${ }^{q}$

P. Borzemski, J. Chwastowski, A. Eskreys, K. Piotrzkowski, M. Zachara, L. Zawiejski

Inst. of Nuclear Physics, Cracow, Poland ${ }^{j}$

L. Adamczyk, B. Bednarek, K. Jeleń, D. Kisielewska, T. Kowalski, M. Przybycień, E. Rulikowska-Zarȩbska, L. Suszycki, J. Zając

Faculty of Physics and Nuclear Techniques, Academy of Mining and Metallurgy, Cracow, Poland ${ }^{j}$

A. Kotański

Jagellonian Univ., Dept. of Physics, Cracow, Poland ${ }^{k}$

L.A.T. Bauerdick, U. Behrens, H. Beier, J.K. Bienlein, C. Coldewey, O. Deppe, K. Desler, G. Drews,

M. Flasiński ${ }^{10}$, D.J. Gilkinson, C. Glasman, P. Göttlicher, J. Große-Knetter, B. Gutjahr ${ }^{11}$, T. Haas, W. Hain, D. Hasell, H. Heßling, Y. Iga, K.F. Johnson ${ }^{12}$, P. Joos, M. Kasemann, R. Klanner, W. Koch, L. Köpke ${ }^{13}$, U. Kötz, H. Kowalski, J. Labs, A. Ladage, B. Löhr, M. Löwe, D. Lüke, J. Mainusch ${ }^{14}$, O. Mańczak, T. Monteiro ${ }^{15}$, J.S.T. Ng, S. Nickel ${ }^{16}$, D. Notz, K. Ohrenberg, M. Roco, M. Rohde, J. Roldán, U. Schneekloth, W. Schulz, F. Selonke, E. Stiliaris ${ }^{17}$, B. Surrow, T. Voß, D. Westphal, G. Wolf, C. Youngman, W. Zeuner, J.F. Zhou ${ }^{18}$

Deutsches Elektronen-Synchrotron DESY, Hamburg, Germany

H.J. Grabosch, A. Kharchilava ${ }^{19}$, A. Leich, S.M. Mari³, M.C.K. Mattingly ${ }^{20}$, A. Meyer,

S. Schlenstedt, N. Wulff

DESY-Zeuthen, Inst. für Hochenergiephysik, Zeuthen, Germany

G. Barbagli, E. Gallo, P. Pelfer

University and INFN, Florence, Italy ${ }^{f}$

G. Maccarrone, S. De Pasquale, L. Votano

INFN, Laboratori Nazionali di Frascati, Frascati, Italy ${ }^{f}$

A. Bamberger, S. Eisenhardt, A. Freidhof, S. Söldner-Rembold ${ }^{21}$, J. Schroeder ${ }^{22}$, T. Trefzger

Fakultät für Physik der Universität Freiburg i.Br., Freiburg i.Br., Germany ${ }^{c}$ 
J.T. Bromley, N.H. Brook, P.J. Bussey, A.T. Doyle, D.H. Saxon, M.L. Utley, A.S. Wilson

Dept. of Physics and Astronomy, University of Glasgow, Glasgow, U.K. ${ }^{\circ}$

A. Dannemann, U. Holm, D. Horstmann, T. Neumann, R. Sinkus, K. Wick

Hamburg University, I. Institute of Exp. Physics, Hamburg, Germany ${ }^{c}$

E. Badura ${ }^{23}$, B.D. Burow ${ }^{24}$, L. Hagge ${ }^{14}$, E. Lohrmann, J. Milewski, M. Nakahata ${ }^{25}$, N. Pavel, G. Poelz, W. Schott, F. Zetsche

Hamburg University, II. Institute of Exp. Physics, Hamburg, Germany ${ }^{c}$

T.C. Bacon, N. Bruemmer, I. Butterworth, V.L. Harris, B.Y.H. Hung, K.R. Long, D.B. Miller, P.P.O. Morawitz, A. Prinias, J.K. Sedgbeer, A.F. Whitfield

Imperial College London, High Energy Nuclear Physics Group, London, U.K. ${ }^{\circ}$

U. Mallik, E. McCliment, M.Z. Wang, S.M. Wang, J.T. Wu

University of Iowa, Physics and Astronomy Dept., Iowa City, USA ${ }^{p}$

P. Cloth, D. Filges

Forschungszentrum Jülich, Institut für Kernphysik, Jülich, Germany

S.H. An, G.H. Cho, B.J. Ko, S.B. Lee, S.W. Nam, H.S. Park, S.K. Park

Korea University, Seoul, Korea ${ }^{h}$

R. Imlay, S. Kartik, H.-J. Kim, R.R. McNeil, W. Metcalf, V.K. Nadendla

Louisiana State University, Dept. of Physics and Astronomy, Baton Rouge, LA, USA ${ }^{p}$

F. Barreiro ${ }^{26}$, G. Cases, J.P. Fernandez, R. Graciani, J.M. Hernández, L. Hervás ${ }^{26}$, L. Labarga ${ }^{26}$, M. Martinez, J. del Peso, J. Puga, J. Terron, J.F. de Trocóniz

Univer. Autónoma Madrid, Depto de Física Teóríca, Madrid, Spain ${ }^{n}$

G.R. Smith

University of Manitoba, Dept. of Physics, Winnipeg, Manitoba, Canada ${ }^{a}$

F. Corriveau, D.S. Hanna, J. Hartmann, L.W. Hung, J.N. Lim, C.G. Matthews ${ }^{27}$, P.M. Patel,

L.E. Sinclair, D.G. Stairs, M. St.Laurent, R. Ullmann, G. Zacek

McGill University, Dept. of Physics, Montréal, Québec, Canada ${ }^{a},{ }^{b}$

V. Bashkirov, B.A. Dolgoshein, A. Stifutkin

Moscow Engineering Physics Institute, Moscow, Russia ${ }^{l}$

G.L. Bashindzhagyan ${ }^{28}$, P.F. Ermolov, L.K. Gladilin, Yu.A. Golubkov, V.D. Kobrin, I.A. Korzhavina,

V.A. Kuzmin, O.Yu. Lukina, A.S. Proskuryakov, A.A. Savin, L.M. Shcheglova, A.N. Solomin,

N.P. Zotov

Moscow State University, Institute of Nuclear Physics, Moscow, Russia ${ }^{m}$

M. Botje, F. Chlebana, A. Dake, J. Engelen, M. de Kamps, P. Kooijman, A. Kruse, H. Tiecke, W. Verkerke, M. Vreeswijk, L. Wiggers, E. de Wolf, R. van Woudenberg ${ }^{29}$

NIKHEF and University of Amsterdam, Netherlands ${ }^{i}$

D. Acosta, B. Bylsma, L.S. Durkin, J. Gilmore, K. Honscheid, C. Li, T.Y. Ling, K.W. McLean ${ }^{30}$, P. Nylander, I.H. Park, T.A. Romanowski ${ }^{31}$, R. Seidlein ${ }^{32}$

Ohio State University, Physics Department, Columbus, Ohio, USA ${ }^{p}$

D.S. Bailey, A. Byrne ${ }^{33}$, R.J. Cashmore, A.M. Cooper-Sarkar, R.C.E. Devenish, N. Harnew,

M. Lancaster, L. Lindemann ${ }^{3}$, J.D. McFall, C. Nath, V.A. Noyes, A. Quadt, J.R. Tickner,

H. Uijterwaal, R. Walczak, D.S. Waters, F.F. Wilson, T. Yip

Department of Physics, University of Oxford, Oxford, U.K. ${ }^{\circ}$

G. Abbiendi, A. Bertolin, R. Brugnera, R. Carlin, F. Dal Corso, M. De Giorgi, U. Dosselli,

S. Limentani, M. Morandin, M. Posocco, L. Stanco, R. Stroili, C. Voci

Dipartimento di Fisica dell' Universita and INFN, Padova, Italy ${ }^{f}$ 
J. Bulmahn, J.M. Butterworth, R.G. Feild, B.Y. Oh, J.R. Okrasinski ${ }^{34}$, J.J. Whitmore

Pennsylvania State University, Dept. of Physics, University Park, PA, USA ${ }^{q}$

G. D'Agostini, G. Marini, A. Nigro, E. Tassi

Dipartimento di Fisica, Univ. 'La Sapienza' and INFN, Rome, Italy ${ }^{f}$

J.C. Hart, N.A. McCubbin, K. Prytz, T.P. Shah, T.L. Short ${ }^{35}$

Rutherford Appleton Laboratory, Chilton, Didcot, Oxon, U.K. ${ }^{\circ}$

E. Barberis, T. Dubbs, C. Heusch, M. Van Hook, W. Lockman, J.T. Rahn, H.F.-W. Sadrozinski, A. Seiden, D.C. Williams

University of California, Santa Cruz, CA, USA ${ }^{p}$

J. Biltzinger, R.J. Seifert, O. Schwarzer, A.H. Walenta, G. Zech

Fachbereich Physik der Universität-Gesamthochschule Siegen, Germany ${ }^{c}$

H. Abramowicz, G. Briskin, S. Dagan ${ }^{36}$, A. Levy ${ }^{28}$

School of Physics,Tel-Aviv University, Tel Aviv, Israel ${ }^{e}$

J.I. Fleck, T. Hasegawa, M. Hazumi, T. Ishii, M. Kuze, S. Mine, Y. Nagasawa, M. Nakao, I. Suzuki, K. Tokushuku, S. Yamada, Y. Yamazaki

Institute for Nuclear Study, University of Tokyo, Tokyo, Japan ${ }^{g}$

M. Chiba, R. Hamatsu, T. Hirose, K. Homma, S. Kitamura, Y. Nakamitsu, K. Yamauchi

Tokyo Metropolitan University, Dept. of Physics, Tokyo, Japan ${ }^{g}$

R. Cirio, M. Costa, M.I. Ferrero, L. Lamberti, S. Maselli, C. Peroni, R. Sacchi, A. Solano, A. Staiano

Universita di Torino, Dipartimento di Fisica Sperimentale and INFN, Torino, Italy ${ }^{f}$

M. Dardo

II Faculty of Sciences, Torino University and INFN - Alessandria, Italy ${ }^{f}$

D.C. Bailey, F. Benard, M. Brkic, G.F. Hartner, K.K. Joo, G.M. Levman, J.F. Martin, R.S. Orr, S. Polenz, C.R. Sampson, R.J. Teuscher

University of Toronto, Dept. of Physics, Toronto, Ont., Canada ${ }^{a}$

C.D. Catterall, T.W. Jones, P.B. Kaziewicz, J.B. Lane, R.L. Saunders, J. Shulman

University College London, Physics and Astronomy Dept., London, U.K. ${ }^{\circ}$

K. Blankenship, B. Lu, L.W. Mo

Virginia Polytechnic Inst. and State University, Physics Dept., Blacksburg, VA, USA ${ }^{q}$

W. Bogusz, K. Charchuła, J. Ciborowski, J. Gajewski, G. Grzelak ${ }^{37}$, M. Kasprzak, M. Krzyżanowski,

K. Muchorowski ${ }^{38}$, R.J. Nowak, J.M. Pawlak, T. Tymieniecka, A.K. Wróblewski, J.A. Zakrzewski, A.F. Żarnecki Warsaw University, Institute of Experimental Physics, Warsaw, Poland ${ }^{j}$

M. Adamus

Institute for Nuclear Studies, Warsaw, Poland ${ }^{j}$

Y. Eisenberg ${ }^{36}$, U. Karshon ${ }^{36}$, D. Revel ${ }^{36}$, D. Zer-Zion

Weizmann Institute, Particle Physics Dept., Rehovot, Israel ${ }^{d}$

I. Ali, W.F. Badgett, B. Behrens ${ }^{39}$, S. Dasu, C. Fordham, C. Foudas, A. Goussiou ${ }^{40}$, R.J. Loveless, D.D. Reeder, S. Silverstein, W.H. Smith, A. Vaiciulis, M. Wodarczyk

University of Wisconsin, Dept. of Physics, Madison, WI, USA ${ }^{p}$

T. Tsurugai

Meiji Gakuin University, Faculty of General Education, Yokohama, Japan

S. Bhadra, M.L. Cardy, C.-P. Fagerstroem, W.R. Frisken, M. Khakzad, W.N. Murray, W.B. Schmidke

York University, Dept. of Physics, North York, Ont., Canada ${ }^{a}$ 
1 also at IROE Florence, Italy

2 now at Univ. of Salerno and INFN Napoli, Italy

${ }^{3}$ supported by EU HCM contract ERB-CHRX-CT93-0376

${ }^{4}$ supported by Worldlab, Lausanne, Switzerland

${ }^{5}$ now a self-employed consultant

${ }^{6}$ now at ELEKLUFT, Bonn

7 now also at University of Torino

8 Alexander von Humboldt Fellow

9 Alfred P. Sloan Foundation Fellow

10 now at Inst. of Computer Science, Jagellonian Univ., Cracow

11 now at Comma-Soft, Bonn

12 visitor from Florida State University

13 now at Univ. of Mainz

${ }^{14}$ now at DESY Computer Center

${ }^{15}$ supported by European Community Program PRAXIS XXI

16 now at Dr. Seidel Informationssysteme, Frankfurt/M.

17 now at Inst. of Accelerating Systems \& Applications (IASA), Athens

18 now at Mercer Management Consulting, Munich

19 now at Univ. de Strasbourg

20 now at Andrews University, Barrien Springs, U.S.A.

${ }^{21}$ now with OPAL Collaboration, Faculty of Physics at Univ. of Freiburg

${ }^{22}$ now at SAS-Institut GmbH, Heidelberg

23 now at GSI Darmstadt

24 also supported by NSERC

${ }^{25}$ now at Institute for Cosmic Ray Research, University of Tokyo

${ }^{26}$ partially supported by CAM

27 now at Park Medical Systems Inc., Lachine, Canada

28 partially supported by DESY

29 now at Philips Natlab, Eindhoven, NL

30 now at Carleton University, Ottawa, Canada

31 now at Department of Energy, Washington

32 now at HEP Div., Argonne National Lab., Argonne, IL, USA

33 now at Oxford Magnet Technology, Eynsham, Oxon

34 in part supported by Argonne National Laboratory

35 now at Digital Equipment International BV., Galway, Ireland

36 supported by a MINERVA Fellowship

37 supported by the Polish State Committee for Scientific Research, grant No. 2P03B09308

38 supported by the Polish State Committee for Scientific Research, grant No. 2P03B09208

39 now at University of Colorado, U.S.A.

${ }^{40}$ now at High Energy Group of State University of New York, Stony Brook, N.Y. 

Collaboration (proj. No. 506/92) (he Polish State Committee for Scientific Research (grant No. 2 P03B 08308 ) partially supported by the German Federal Ministry for Education and Science, Research and Technology (BMBF)

supported by the German Federal Ministry for Education and Science, Research and Technology (BMBF), and the Fund of Fundamental Research of Russian Ministry of Science and Education and by INTAS-Grant No. 93-63

$n$ supported by the Spanish Ministry of Education and Science through funds provided by CICYT

o supported by the Particle Physics and Astronomy Research Council

$p \quad$ supported by the US Department of Energy 


\section{Introduction}

Inclusive particle distributions have been widely studied in deep inelastic scattering (DIS) [1] and $e^{+} e^{-}$annihilation to investigate the nature of the quark fragmentation and effects of higher order QCD processes. The formation of hadrons in DIS is a complicated process which cannot be fully calculated in the framework of perturbative QCD. In order to model this process it is convenient to distinguish two phases of the hadron formation. These correspond to a perturbative phase for QCD processes on the parton level followed by a non-perturbative fragmentation phase describing the confinement of the partons to observable hadrons.

In this paper the charged hadron multiplicity distributions are analysed in the virtual-photon proton centre-of-mass system $\left(\gamma^{*} p \mathrm{cms}\right)$, which corresponds to the centre-of-mass system of the produced hadronic final state with the invariant mass $W$. In the naive quark parton model (QPM) the virtual photon hits a quark in the proton and transfers a four momentum, $q$. The struck quark and the target remnant system each have an energy of $W / 2$ in the $\gamma^{*} p$ cms and move back-to-back with a 'velocity', which corresponds to a rapidity $y_{\text {max }}$ proportional to $\pm \ln W$. The outgoing quark and target remnant hadronise into multi-particle final states with limited $p_{t}^{*}$, where $p_{t}^{*}$ is the hadron momentum component transverse to the virtual photon direction as measured in the $\gamma^{*} p$ cms. The width of the rapidity distributions of the produced hadrons is proportional to $\ln W$, while its height is approximately independent of $W$. From the measurement of jet profiles in DIS it is known that the width of a quark jet is typically two units of rapidity [2]. At high values of $W$, the rapidity range populated by hadrons can be divided into three regions: the current jet region from $\left(y_{\max }-2\right)$ to $y_{\max }$, the region of the target remnant fragmentation from $-y_{\max }$ to $\left(-y_{\max }+2\right)$ and a plateau region in between. When analysing hadron distributions as a function of the scaled longitudinal momentum in the $\gamma^{*} p$ cms, $x_{F}$, the current jet region defined above corresponds to the $x_{F}$ range $x_{F}>0.05$. If no QCD branching processes on the parton level are considered, the $x_{F}$ and $p_{t}^{*}$ distributions for $x_{F}>0.05$ are predicted to scale in $W$.

In fixed target DIS experiments [3, 4, 5] effects of scale-breaking in the $x_{F}$ distributions from QCD corrections, which are expected to soften the observed spectrum with increasing $W$, are small and could not be unambiguously identified. On the other hand, the mean square of $p_{t}^{*}$, $\left\langle p_{t}^{* 2}\right\rangle$, has been found to be very sensitive to higher order QCD effects [6]. However, the details of the $p_{t}^{*}$ spectra are also sensitive to non-perturbative fragmentation effects [5, 7, 8]. With the high energies reached in ep collisions at HERA it is possible to extend the studies of $x_{F}$ and $p_{t}^{*}$ distributions to larger values of $W$, where the influence of perturbative QCD effects is expected to be much larger and the final state hadron distributions should reflect the dynamics of the subprocesses on the parton level.

In a recent analysis the scaled momentum and charged multiplicity distributions of the hadronic final state were measured in the current region of the Breit frame as a function of the negative square of the four-momentum transfer, $Q^{2}$, and the Bjorken-scaling variable $x$ [9]. The charged particle spectra were observed to evolve with $Q^{2}$ in a way similar as in $e^{+} e^{-}$annihilation. In this paper we study inclusive charged hadron production as a function of $x_{F}$ and $<p_{t}^{* 2}>$ in the current region of the $\gamma^{*} p$ cms frame. The objective of the analysis is to investigate the influence of perturbative QCD effects on the hadronic final state by studying the $W$ dependence of these distributions in HERA ep collisions and in fixed target DIS data. The data are also compared

\footnotetext{
${ }^{1}$ The rapidity is measured with respect to the virtual photon in the $\gamma^{*} p \mathrm{cms}$.
} 
with $e^{+} e^{-}$results as well as with predictions of Monte Carlo programs. The comparison is also performed for a subclass of DIS events, which are characterised by a rapidity gap between the observed hadronic final state and the proton beam direction [10], and which are therefore candidates for diffractive scattering.

\section{The experiment}

\subsection{HERA}

The data were collected during the 1993 running period using the ZEUS detector at the electronproton collider HERA, where a $26.7 \mathrm{GeV}$ electron beam and a $820 \mathrm{GeV}$ proton beam were brought to collision providing an ep centre-of-mass energy of $296 \mathrm{GeV}$. 84 bunches were filled for each beam and in addition 10 electron and 6 proton bunches were left unpaired for background studies. An integrated luminosity of $0.55 \mathrm{pb}^{-1}$ was collected.

\subsection{The ZEUS detector}

ZEUS is a multi-purpose magnetic detector which has been described elsewhere [11, 12]. Here a brief description is given which concentrates on those parts of the detector relevant for the present analysis.

Charged particles are tracked by the inner tracking detectors which operate in a magnetic field of $1.43 \mathrm{~T}$ provided by a thin superconducting solenoid. Immediately surrounding the beam pipe is the vertex detector (VXD) which consists of 120 radial cells, each with 12 sense wires 13 . The achieved resolution is $50 \mu \mathrm{m}$ in the central region of a cell and $150 \mu \mathrm{m}$ near the edges. Surrounding the VXD is the central tracking detector (CTD) which consists of 72 cylindrical drift chamber layers, organised into 9 'superlayers' [14]. These superlayers alternate between those with wires parallel (axial) to the collision axis and those inclined at a small angle to give a stereo view. The hit efficiency of the CTD is greater than $95 \%$ and the resolution in transverse momentum for full length tracks is $\sigma_{p_{T}} / p_{T}=0.005 p_{T} \otimes 0.016\left(p_{T}\right.$ in $\left.\mathrm{GeV}\right)$, where $\otimes$ means addition in quadrature.

The solenoid is surrounded by a high resolution uranium-scintillator calorimeter (CAL), which is divided into three parts: forward (FCAL), barrel (BCAL) and rear (RCAL) 15]. It covers $99.7 \%$ of the solid angle. Holes of $20 \times 20 \mathrm{~cm}^{2}$ in the centre of FCAL and RCAL accommodate the HERA beam pipe. Each of the calorimeter parts is subdivided into towers which in turn are segmented longitudinally into electromagnetic (EMC) and hadronic (HAC) sections. These sections are further subdivided into cells, which are read out by two phototubes each.

For measuring the luminosity as well as for tagging very small $Q^{2}$ processes, two lead-scintillator calorimeters are used [12, 16]. Bremsstrahlung photons emerging from the electron-proton interaction point (IP) at angles $\theta_{\gamma} \leq 0.5 \mathrm{mrad}$ with respect to the electron beam axis hit the photon calorimeter at $107 \mathrm{~m}$ from the IP. Electrons emitted from the IP at scattering angles less than 6 mrad and with energies between $20 \%$ and $90 \%$ of the nominal beam energy are deflected by beam magnets and hit the electron calorimeter placed $35 \mathrm{~m}$ from the IP.

\footnotetext{
${ }^{2}$ The proton beam direction is the forward $+Z$ direction.
} 
Two small lead-scintillator sandwich counters partially surround the beam-pipe at the rear of the RCAL. These counters were used to reject background produced by beam-gas interactions with the incoming proton beam and to measure the timing and longitudinal spread of both the proton and the electron beams of HERA. Two layers of scintillation counters mounted on either side of an iron veto wall, situated upstream of the detector, were also used to reject background particles.

\section{$3 \quad$ Data taking conditions}

The ZEUS trigger is organised in three levels $[11]$ and reduces the input event rate from the bunch crossing rate of $10 \mathrm{MHz}$ to $3-5 \mathrm{~Hz}$. For DIS events, the first level trigger (FLT) requires at least one of three conditions for energy sums in the EMC calorimeter cells: the BCAL EMC energy exceeds $3.4 \mathrm{GeV}$; or the RCAL EMC energy (excluding the innermost towers surrounding the beam pipe) exceeds $2.0 \mathrm{GeV}$; or the RCAL EMC energy (including those towers) exceeds $3.75 \mathrm{GeV}$.

The second level trigger (SLT) rejects proton beam-gas events by using the event times measured in the rear calorimeter cells. The DIS trigger rate of the SLT is about one-tenth the FLT DIS trigger rate. The loss of DIS events at the SLT is negligible.

The third level trigger (TLT) has the full event information available and applies physics-based filters. It requires tighter timing cuts to suppress beam-gas background further and also rejects beam halo muons and cosmic muons. The TLT selects DIS event candidates by calculating:

$$
\delta=\sum_{i} E_{i} \cdot\left(1-\cos \theta_{i}\right) \quad>20 \mathrm{GeV}-2 E_{\gamma}
$$

where $E_{i}$ and $\theta_{i}$ are the energy and the polar angle of the energy deposits in the calorimeter. The summation runs over all calorimeter cells. $E_{\gamma}$ is the energy measured in the photon calorimeter of the luminosity monitor. For fully contained DIS events $\delta \approx 2 E_{e}=53.4 \mathrm{GeV}$, where $E_{e}$ is the energy of the incident electron. Photoproduction events have low values of $\delta$ compared to DIS events because the scattered electron escapes in the hole of the calorimeter which contains the beam pipe.

For events with the scattered electron detected in the calorimeter, the trigger is essentially independent of the DIS hadronic final state. The trigger acceptance is greater than $97 \%$ for $Q^{2}>10 \mathrm{GeV}^{2}$ and independent of $Q^{2}$ [17]. A total of about $7 \cdot 10^{6}$ events passed the TLT and was written to tape during the 1993 running period.

\section{Event kinematics}

In deep inelastic ep scattering events the incoming electron couples to a $\gamma$ or a $Z$ (neutral current $\mathrm{NC}$ ) or to a $W^{+}$(charged current CC), which scatters off the proton. In the $Q^{2}$ range explored here, the contribution from $W$ and $Z$ exchange is negligible. The kinematic variables used to describe the inclusive DIS process are defined in Table 1 .

\footnotetext{
${ }^{3}$ The proton beam direction is defined as the $Z$-axis in the HERA laboratory frame.
} 


\begin{tabular}{|l|l|}
\hline \multicolumn{1}{|c|}{ Variable } & \multicolumn{1}{c|}{ Description } \\
\hline$l\left(l^{\prime}\right)$ & Four-momentum of the incident (scattered) lepton \\
$P, M_{p}$ & Four-momentum of the proton and its mass \\
$Q^{2}=-q^{2}=-\left(l-l^{\prime}\right)^{2}$ & Negative invariant mass squared of the exchanged virtual \\
& boson \\
$\begin{array}{l}\nu=(P \cdot q) / M_{p} \\
x=Q^{2} /(2 P \cdot q)\end{array}$ & Energy of the exchanged boson in the proton rest frame \\
$=Q^{2} /\left(2 M_{p} \nu\right)$ & Bjorken scaling variable \\
$y=(P \cdot q) /(P \cdot l)$ & Inelasticity parameter \\
$W^{2}=(P+q)^{2}$ & Invariant mass squared of the hadronic final state \\
$=Q^{2}(1-x) / x+M_{p}^{2}$ & \\
\hline
\end{tabular}

Table 1: Definition of the variables used to describe the kinematics of the inclusive DIS process

The ZEUS detector is almost hermetic, allowing the kinematic variables $Q^{2}, x$ and $y$ to be reconstructed in a variety of ways using combinations of electron and hadronic system energies and angles. In the analysis presented here the double angle method (DA) was chosen, in which the scattered electron angle and the angle $\gamma_{H}$ is used [18. In the naive quark parton model $\gamma_{H}$ corresponds to the angle of the scattered massless quark in the laboratory frame. The variable $y$ is determined according to the Jacquet-Blondel method [19 and is denoted by $y_{J B}$.

The four-momentum of the scattered electron needed to calculate the Lorentz boost to the $\gamma^{*} p$ cms frame, is reconstructed from its polar and azimuthal angle, $\theta_{e}, \phi_{e}$. The scattered electron energy $E_{D A}^{\prime}$, used in the boost, is computed by the double angle method:

$$
E_{D A}^{\prime}=Q_{D A}^{2} /\left(2 E_{e}\left(1+\cos \theta_{e}\right)\right)
$$

where $E_{e}$ is the energy of the incident electron and $Q_{D A}^{2}$ is given by:

$$
Q_{D A}^{2}=4 E_{e}^{2} \cdot \frac{\sin \gamma_{H}\left(1+\cos \theta_{e}\right)}{\sin \gamma_{H}+\sin \theta_{e}-\sin \left(\gamma_{H}+\theta_{e}\right)} .
$$

The variables $x_{F}$ and $p_{t}^{*}$ describe the kinematics of the hadrons in the $\gamma^{*} p$ cms:

$$
x_{F}=p_{l}^{*} /\left|p_{l, \max }^{*}\right|=2 p_{l}^{*} / W
$$

where $p_{l}^{*}$ is the projection of the hadron momentum vector onto the direction of the virtual photon and $\left|p_{l, \text { max }}^{*}\right|$ is the maximum value of $p_{l}^{*}$. The hadron momentum component perpendicular to the virtual photon axis is denoted by $p_{t}^{*}$.

\section{$5 \quad$ Data selection}

\subsection{Event selection}

The offline selection of DIS events was similar to that described in earlier publications (e.g. [9, 20, 21]). Scattered electron candidates were selected by using the pattern of energy deposition in the calorimeter. The electron identification algorithm was tuned for purity 
rather than for efficiency. The purity is defined as the number of electrons generated and reconstructed in a bin divided by the total number of electron candidates measured in the bin. In studies with Monte Carlo DIS events and test beam data the purity was estimated to be $\geq 96 \%$ for $E_{D A}^{\prime} \geq 10 \mathrm{GeV}$.

The requirements for the final event selection were:

- $E_{D A}^{\prime} \geq 10 \mathrm{GeV}$, to minimise beam gas background contamination;

- $Q_{D A}^{2} \geq 10 \mathrm{GeV}^{2}$

- $y_{e} \leq 0.85$, to reduce the photoproduction background, where $y_{e}$ is the scaling variable $y$ as determined from the energy and polar angle of the scattered electron;

- $y_{J B} \geq 0.04$, to guarantee sufficient accuracy for the $D A$ reconstruction method;

- $\delta=\sum_{i} E_{i}\left(1-\cos \theta_{i}\right) \geq 35 \mathrm{GeV}$, where the sum runs over all calorimeter cells. For fully contained events $\delta \approx 2 E_{e}=53.4 \mathrm{GeV}$. This cut is used to remove photoproduction events and to control radiative corrections.

Furthermore we required:

- a primary vertex position, determined from VXD and CTD tracks, in the range $-50 \leq Z_{v t x} \leq 40 \mathrm{~cm}$

- the impact point $(X, Y)$ of the scattered electron in the RCAL to lie outside a square of $32 \times 32 \mathrm{~cm}^{2}$ centered on the beam axis, to ensure that the electron is fully contained within the detector and its position can be reconstructed with sufficient accuracy.

After these cuts, the remaining photoproduction background was estimated to be $\simeq 1 \%$. The contamination from beam-gas background was estimated to be below $0.5 \%$ as calculated from unpaired electron and proton bunches. Finally, QED Compton scattering events and residual cosmic and beam-related muons were rejected by algorithms, which identify this types of events by their pattern of energy deposits in the calorimeter cells.

A total of 26100 events was selected by the above cuts. Of these events about 10\% [10] contain a large rapidity gap in the hadronic final state. They are characterised by $\eta_{\max }<1.5$, where $\eta_{\max }$ is the pseudorapidity of the most forward calorimeter cluster in the event, relative to the proton direction. The pseudorapidity is defined by $\eta=-\ln (\tan (\theta / 2))$ and a cluster is an isolated set of adjacent calorimeter cells with summed energy above $400 \mathrm{MeV}$. This sample is called the 'large-rapidity-gap' (LRG) event sample. The remaining events are denoted by 'non-rapidity-gap' (NRG) events. The invariant mass of the hadronic final state excluding the scattered proton in the LRG events is calculated from the energy deposits measured in the calorimeter (excluding the electron cluster) by $M_{X}=\sqrt{\sum_{h a d}\left(E^{2}-p_{X}^{2}-p_{Y}^{2}-p_{Z}^{2}\right)}$. The values of $p_{X}, p_{Y}$ and $p_{Z}$ are the cell energies $E$ projected on the axes of the HERA laboratory frame. The polar angles of these pseudovectors are calculated from the geometric centres of the cells and the primary event vertex position. The measured value of $M_{X}$ is corrected to the hadron level as described in section 6 . 


\subsection{Track reconstruction and selection}

Tracks were recognised and fitted using two programs which were developed independently and follow different strategies for pattern recognition and track fitting. For the results shown in this paper the first approach is used and the second method was used for estimating the systematic error.

In the first approach the track finding algorithm starts with hits in the outermost axial superlayers of the CTD. As the trajectory is followed inwards to the beam axis, more hits from the axial wires of the CDT and of the VXD are incorporated. A circle is fitted in the $X Y$ projection and is used for the pattern recognition in the stereo superlayer pattern. The momentum vector is determined in a 5-parameter helix fit.

The second track finding program is based on the Kalman filtering technique [22]. Seed tracks found in the outer layers of the CTD are extended inwards and points are added as wire layers of the CTD are crossed. The track parameters at each step are updated using the Kalman method. In the second step a Kalman fit to the points found in the pattern recognition phase is performed taking into account non-linear corrections to the measured drift time. Following the reconstructed CTD track inwards, CTD and VXD hits are associated with the track. The VXD track segments are merged with the CTD tracks using the Kalman filtering algorithm.

Multiple Coulomb scattering in the beam pipe and in the walls of the VXD and CTD were taken into account in the evaluation of the covariance matrix. The vertex fit is performed with the fitted tracks using the perigee parameterisation [23]. The vertex position is evaluated and the track parameters are calculated at the vertex.

Only tracks which are associated with the primary vertex have been selected for this analysis. The tracks are required to have $p_{t, l a b}>0.2 \mathrm{GeV}$ and a polar angle in the HERA laboratory frame in the range of $25^{\circ}<\theta<155^{\circ}$. This is a region of the CTD, where the detector response and systematics are best understood. For tracks defined by these cuts the track reconstruction efficiency is $\simeq 95 \%$.

The scattered electron was removed from the track sample by rejecting those tracks which match the cluster in the calorimeter assigned to the scattered electron by the electron finding algorithm. Only tracks which reach at least the third superlayer and hence have a projected length in the plane perpendicular to the beam axis of more than $30 \mathrm{~cm}$ are kept to achieve the required transverse momentum resolution. For $\theta>150^{\circ}$ the efficiency for identifying the scattered electron by matching the CTD tracks to energy deposits in the calorimeter decreases rapidly due to the limited acceptance and resolution of the CTD in the very rear part of the detector. Therefore the upper cut on $\theta$ of the hadrons considered in the analysis was further tightened to $150^{\circ}$.

Due to the cuts in $\theta$ and $p_{t, l a b}$ the analysis in the $\gamma^{*} p$ cms is restricted to the range $10<Q^{2}<$ $160 \mathrm{GeV}^{2}$ and $75<W<175 \mathrm{GeV}$, where the acceptance for charged hadrons is larger than $60 \%$.

In Fig. [1 the distribution of the selected events in the $Q^{2}-x$ plane is shown. For comparison the kinematic region which has been investigated in fixed target experiments is also shown. 


\section{Acceptance correction}

\subsection{Monte Carlo simulation}

The measured distributions are corrected for detector effects such as acceptance and resolution. For that purpose the hadronic final state from DIS was modelled using two different sets of Monte Carlo generators, the first for the description of the non-rapidity-gap events and the second to model the large-rapidity-gap events.

Events from NRG DIS processes were generated using two alternative Monte Carlo models: a) the combination of the LEPTO 6.1 [24] and the ARIADNE 4.0 Monte Carlo program [25, 26] (CDMBGF) and b) LEPTO 6.1 with the option of combined matrix element and parton shower calculation (MEPS). The fragmentation was simulated using the LUND string model 27 as implemented in JETSET [28] (see Table 2).

Both models were interfaced to the program HERACLES [29], which computes the electroweak radiative corrections for DIS events. In the case of hard QED Bremsstrahlung the fourmomentum vector of the virtual photon which probes the proton is significantly different from the virtual photon momentum reconstructed from the momenta of the incident and scattered lepton. In this case the $x_{F}$ and $p_{t}^{*}$ distributions are also distorted and have to be corrected for this effect. In this analysis, however, the virtual photon momentum was reconstructed using the double angle method, which is insensitive to radiative effects. Events with hard QED initial state Bremsstrahlung photons $\left(E_{\text {brems }} \gtrsim 7 \mathrm{GeV}\right.$ ) are rejected by the cut on $\delta>35 \mathrm{GeV}$ (see section 5.1). Monte Carlo calculations show that the QED radiative corrections are $5-10 \%$.

For both Monte Carlo simulations the $M R S D_{-}^{\prime}$ parameterisation of the parton densities in the proton was chosen [30], which gives a reasonable description of the structure function measured at HERA [31, 32].

The properties of LRG events are characteristic of diffractive interactions [10]. Two Monte Carlo event samples have been used to model the hadronic final state of LRG events. The first was generated using the POMPYT Monte Carlo program [33], which is based on a factorisable model for high energy diffractive processes. Within the PYTHIA [34 framework, the incident proton emits a pomeron, whose constituents take part in a hard scattering process with the virtual photon or its constituents. The structure of the pomeron is assumed to be described by either a hard or a soft quark density function $f(\beta)$, where $\beta$ denotes the fraction of the pomeron momentum carried by the quark.

The second sample was generated following the model of Nikolaev and Zakharov (NZ) 355, which was interfaced to the Lund fragmentation scheme [36]. In the NZ model it is assumed that the exchanged virtual photon fluctuates into a $q \bar{q}$ pair, which interacts with a colourless two-gluon system emitted by the incident proton. Both diffractive Monte Carlo samples were generated with default parameter settings. QED radiative processes were not simulated for these events. With the event selection cuts described in section 5, however, the QED radiative corrections are expected to be of the same size as for the NRG events.

Event samples produced by the Monte Carlo generators marked in Table 2 by an asterisk were also processed by the ZEUS detector simulation program, which is based on GEANT 3.13 [37] and which incorporates the detector and trigger simulation. Events fulfilling the trigger conditions were then passed through the standard ZEUS offline reconstruction program. 


\begin{tabular}{|c|c|}
\hline Acronym & Description \\
\hline $\mathrm{QPM}$ & Quark parton model + string fragmentation only \\
\hline $\mathrm{CDM}$ & Colour dipole model [25, 26] \\
\hline $\operatorname{MEPS}(*)$ & $\begin{array}{l}\text { Parton shower [24] matched to complete } \mathcal{O}\left(\alpha_{s}\right) \text { matrix element } \\
\text { calculation (ME) }\end{array}$ \\
\hline CDMBGF $(*)$ & $\begin{array}{l}\text { Colour dipole model combined with complete } \mathcal{O}\left(\alpha_{s}\right) \text { matrix element } \\
\text { calculation for the BGF process }(\mathrm{ME})\end{array}$ \\
\hline $\operatorname{POMPYT}(*)$ & $\begin{array}{l}\text { Model for diffractive DIS (assuming factorisation of the pomeron } \\
\text { flux and the pomeron structure function) [33] with } \\
\text { a hard quark density function for the pomeron } \propto[\beta(1-\beta)] \\
\text { or a soft quark density function for the pomeron } \propto\left[(1-\beta)^{5}\right] \\
\text { Model for diffractive DIS (non factorisable ansatz) }[35]\end{array}$ \\
\hline $\mathrm{NZ}(*)$ & Model for diffractive DIS (non factorisable ansatz) [35] \\
\hline
\end{tabular}

Table 2: Acronyms for the DIS models used in this report. For those generators marked by an asterisk, event samples have also been processed by the detector simulation and data reconstruction program. In all models the LUND string fragmentation model is used [27, 28].

The predicted $\eta_{\max }$ distribution for non-diffractive DIS events falls exponentially for $\eta_{\max }<4$, whereas for diffractive events this distribution is approximately flat. Calculations with the CDMBGF Monte Carlo model show that the fraction of non-diffractive DIS events with $\eta_{\max }<$ 1.5 is about 5\% [10]. The distributions for the LRG event sample defined by $\eta_{\max }<1.5$ have been corrected with POMPYT and those for the NRG events have been corrected using the CDMBGF Monte Carlo program interfaced to HERACLES. Note that the results are not corrected for the selection inefficiency of the $\eta_{\max }$ cut.

\subsection{Data correction procedure}

The measured hadron multiplicity distributions are distorted with respect to those of the true hadronic final state due to trigger biases, event and track selection cuts and the acceptance and resolution of the detector. The output of the trigger and detector simulation program together with the samples produced by the different event generators have been used to estimate the distortion of the distributions and to correct for them by multiplying the measured distributions by a correction function $c(v)$ in each bin of $Q^{2}$ and $W$, where $v$ is the hadron variable under study and $c(v)$ is calculated as a bin-by-bin ratio:

$$
c(v)=\left(\frac{1}{N_{e v t}} \frac{\Delta N_{h a d}(v)}{\Delta v}\right)_{g e n} /\left(\frac{1}{N_{e v t}} \frac{\Delta N_{h a d}(v)}{\Delta v}\right)_{r e c} .
$$

The subscripts gen and rec refer to the quantities as given by the event generator programs and the reconstructed quantities from the output of the detector simulation program, respectively. The number of events in a bin of $Q^{2}$ and $W$ is denoted by $N_{e v t} ; \Delta N_{\text {had }}$ is the number of hadrons in a bin of $v$. The generated hadron distributions do not include the charged particle decay products of $K^{0}$ 's and $\Lambda$ 's and of weakly decaying particles with a lifetime $>10^{-8} \mathrm{~s}$. For the expression in the numerator events and hadrons are sorted in bins of the generated kinematic variables and for the denominator in bins of the reconstructed variables. In this way the 
distributions have been corrected for losses of events and hadrons as well as for the effects of event migration, finite resolution and trigger biases.

The bin size in the hadron variables $v$ was chosen to be comparable with the estimated resolution in $v$ and it was checked that the correction factor neither deviates by more than $40 \%$ from unity nor depends strongly on $v$ [38]. For models which adequately describe the data, the dependence of the correction factors on the model input was found to be small. The difference in $c(v)$ for different models was included in the systematic error.

The mean square of $p_{t}^{*}\left(<p_{t}^{* 2}>\right)$ was corrected by:

$$
<p_{t}^{* 2}>=<p_{t}^{* 2}>_{\text {meas }} \frac{<p_{t}^{* 2}>_{M C, g e n}}{<p_{t}^{* 2}>_{M C, \text { rec }}},
$$

where $\left\langle p_{t}^{* 2}>_{\text {meas }}\right.$ is the mean value of $p_{t}^{* 2}$ determined from the uncorrected data. The terms in the correction factor are defined as in equation 4 . This method of correction is numerically more stable than the determination of $<p_{t}^{* 2}>$ from the acceptance corrected $p_{t}^{* 2}$ distributions.

The following sources of systematic uncertainties were studied:

- The model dependence of the correction factors $c(v)$ was estimated using two different models for the NRG and LRG event samples each. The CDMBGF and MEPS models were used to correct the NRG event sample and for the LRG event sample the POMPYT model with a hard quark density function (see Table 2) and the NZ model were used. The relative systematic error of $1 / N_{\text {evt }} \cdot d N_{h a d} / d x_{F}$ is $\sim 3 \%$ and the one of $1 / N_{\text {evt }} \cdot d N_{\text {had }} / d<p_{t}^{* 2}>$ is $\sim 7 \%$.

- The analysis was done using two different strategies for track finding and vertex fitting as described in section 5.2. The difference of the corrected $x_{F}$ and $p_{t}^{*}$ distributions obtained with both programs is used as an estimate of the systematic error from the track reconstruction. The relative systematic error of $1 / N_{\text {evt }} \cdot d N_{\text {had }} / d x_{F}$ is $\sim 10 \%$ and the one of $1 / N_{\text {evt }} \cdot d N_{\text {had }} / d<p_{t}^{* 2}>$ is $\sim 4 \%$.

- Systematic uncertainties in the determination of the four-momentum of the virtual photon may induce a systematic error in the hadron distributions measured as a function of $x_{F}$ and $p_{t}^{*}$. The size of this systematic error was estimated from Monte Carlo events by using the generated four-momentum of the virtual photon rather than the reconstructed four-momentum. The Lorentz transformation with the generated values was then used to calculate the momenta of the reconstructed final state particles in the $\gamma^{*} p \mathrm{cms}$ and these values were compared to those obtained via the reconstructed virtual photon momentum. The relative systematic error of $1 / N_{\text {evt }} \cdot d N_{\text {had }} / d x_{F}$ is $\sim 7 \%$ and the one of $1 / N_{\text {evt }}$. $d N_{\text {had }} / d<p_{t}^{* 2}>$ is $\sim 5 \%$.

- The sensitivity of the measurements on the track selection criteria has been investigated. The cut in the polar angle of the tracks was varied between $20^{\circ}$ and $33^{\circ}$ and/or it was required that superlayer 5 instead of superlayer 3 has to be reached by the track. The requirement of a minimum hadron momentum transverse to the beam direction in the laboratory frame, $p_{t, l a b}$, was omitted. No significant changes in the results $(<1 \%)$ have been observed. 
- The effect of a possible misestimation of the momentum resolution in the detector simulation program was studied by evaluating the correction function with a resolution of the measured transverse momentum artificially increased by $100 \%$. The size of this effect on $<p_{t}^{* 2}>$ and $x_{F}$ was smaller than $1 \%$.

The contributions of the above effects to the systematic error have been added in quadrature and are shown together with the statistical errors of the results in the tables and figures.

The shape of the correction factors to be applied to the measured hadron distributions of $x_{F}$ and $p_{t}^{*}$ as well as to $\left\langle p_{t}^{* 2}\right\rangle$ is shown in Fig. 2 separately for NRG and LRG events. The size of the correction for both event classes is very similar.

\section{$7 \quad$ Results}

\section{1 $x_{F}$ and $p_{t}^{*}$ distributions in NRG events}

First the $x_{F}$ and $p_{t}^{*}$ distributions of charged hadrons in NRG events are discussed. In Fig. [3a the $x_{F}$ distribution at $\langle W\rangle=120 \mathrm{GeV}$ and $\left.<Q^{2}\right\rangle=28 \mathrm{GeV}^{2}$ is compared with different models for hadron production in DIS. The $x_{F}$ distribution falls steeply with increasing $x_{F}$. The results from the $\mathrm{H} 1$ experiment [39] agree well with this measurements. The data agree with those models, in which higher order QCD processes are included, such as MEPS (solid line) and CDMBGF (dashed line), but not with the naive quark parton model (QPM) (dotted line).

In Fig. $3 \mathrm{~b}$ the $p_{t}^{*}$ spectrum, which is integrated over $x_{F}>0.05$ for the study of the current jet fragmentation, is compared with the same model calculations. The QPM model predicts a much steeper $p_{t}^{*}$ distribution than the data show, whereas the MEPS model agrees well with the data. However, for closer investigation it is advantageous to take the mean square of $p_{t}^{*}$, $\left\langle p_{t}^{* 2}\right\rangle$, a quantity which is more sensitive to the behaviour of the tail of the $p_{t}^{* 2}$ distribution.

Figure Ec shows the $\left\langle p_{t}^{* 2}\right\rangle$ distribution as a function of $x_{F}$ for $x_{F} \geq 0.05$. In any model, which allows for a transverse momentum of the partons, the rise of $\left\langle p_{t}^{* 2}\right\rangle$ with increasing $x_{F}$ is expected because a hadron with a higher value of $x_{F}$ carries also a larger fraction of the transverse momentum of the primary parton. Again the MEPS and CDMBGF models describe the data while the QPM strongly underestimates the value of $\left\langle p_{t}^{* 2}\right\rangle$.

In Fig. [a,c the results from the H1 experiment are also shown [39]. The differential hadron multiplicities measured by ZEUS are listed in Table 3 .

\section{2 $x_{F}$ and $p_{t}^{*}$ spectra in LRG events}

The $x_{F}$ and $p_{t}^{*}$ distributions from charged hadrons as well as $\left\langle p_{t}^{* 2}\right\rangle$ as a function of $x_{F}$ are shown in Fig. 0 separately for the samples of LRG and NRG events. The values for the LRG events are tabulated in Table [4. The value of $\langle W\rangle$ is similar for both event samples, whereas $<Q^{2}>$ for the LRG events is lower by $30 \%$ than for the NRG events. The $x_{F}$ distribution for the LRG events is falling less steeply when compared to that of the NRG events. The LRG data in Fig. 1 are reasonably well described by the POMPYT (solid line) and the NZ (dashed 
line) models for diffractive DIS with the $\eta_{\max }$ cut applied. The QPM prediction for the $x_{F}$ distribution of DIS events, shown by the dotted line in Fig. 4a, is slightly steeper than the $x_{F}$ distribution for LRG events.

The $p_{t}^{*}$ spectrum of LRG events is significantly less broad than that for the rest of the DIS events (Fig. Gb). This effect is highlighted in Fig. 4c. The mean values of $p_{t}^{* 2}$ in events with a large rapidity gap are smaller than for the NRG events by a factor of $2-5$. From a comparison with DIS model calculations with and without simulating QCD radiation processes, it is found that the $<p_{t}^{* 2}>$ values for LRG events resemble those for DIS events with only a small amount of gluon radiation. This observation is in good agreement with ZEUS results from the analysis of the energy flow [21]. However, $\left\langle p_{t}^{* 2}>\right.$ in LRG events is somewhat larger than predicted by the QPM (see dotted line in Fig. \&c), indicating that there is a non-zero contribution of higher order QCD processes in this class of events, too. This is confirmed by the observation of DIS events with a large rapidity gap which exhibit a two-jet structure [40]. The model calculations for diffractive $e p$ scattering slightly underestimate the measured values of $\left\langle p_{t}^{* 2}\right\rangle$.

The inclusive distributions of LRG events have been found to have the properties of a diffractive interaction of a highly virtual photon with a proton [10]. Diffractive interactions in hadronhadron reactions and photoproduction have been successfully described in the framework of Regge theory by the exchange of a pomeron [41. Several models have been developed to describe this reaction in terms of parton interactions (e.g. [42, 43]). In this context it is interesting to test the hypothesis that the diffractive DIS process can be viewed as the 'emission' of a pomeron from the proton, which carries the fraction $x_{\text {pom }}$ of the proton momentum, and a subsequent deep inelastic $\gamma^{*}$ pomeron scattering, which occurs at a higher value of $x^{\prime}=\frac{x}{x_{\text {pom }}}$. In this picture the relevant scale for the invariant mass of the hadronic final state should be given by $M_{X}$ and not by $W$. In Fig. $5 \mathrm{a}<p_{t}^{* 2}>$ as a function of $x_{F}$ from the LRG events is compared with the results of a fixed target DIS experiment [5], where the invariant mass of the total hadronic final state $(\langle W\rangle=14 \mathrm{GeV})$ is only slightly higher than the invariant mass of the hadronic final state observed in the LRG events $\left(\left\langle M_{X}\right\rangle=8 \mathrm{GeV}\right)$. The values of $\left\langle p_{t}^{* 2}\right\rangle$ and the $x_{F}$ distribution for both event samples agree reasonably well. This result supports the hypothesis that the transverse momentum space for the particle production is similar to DIS, where the scale of the invariant mass is given by $M_{X}$ rather than by $W$.

\section{3 $W$ and $Q^{2}$ dependence of $x_{F}$ and $p_{t}^{*}$ spectra}

In Fig. 6a the $x_{F}$ distribution from the NRG events is compared with that from $e^{+} e^{-}$annihilation events on the $Z^{0}$ resonance [44], where the value of the $e^{+} e^{-}$centre-of-mass energy is comparable to the value of $W$ in the kinematic range analysed here. The differential rates for hadron production in $e^{+} e^{-}$annihilation were divided by two so that they correspond to a single hemisphere and can be directly compared with the results from DIS. The differential hadron multiplicity distribution in DIS at HERA energies agrees with that observed in $e^{+} e^{-}$ collision events for $x_{F} \gtrsim 0.1$. This confirms the approximate independence of the hadron formation process from the type of the primary scattering objects, which most of the models assume [27, 45.

The $x_{F}$ and $<p_{t}^{* 2}>$ distributions from this analysis are compared with those of DIS events at lower values of $W$ [46, 47]. Since in fixed target experiments the DIS event sample has not been separated into NRG and LRG events, the NRG and LRG event samples have been combined 
for the comparison. The $x_{F}$ and $p_{t}^{*}$ distribution as well as $\left\langle p_{t}^{* 2}\right\rangle$ as a function of $x_{F}$ for the $\mathrm{NRG}+\mathrm{LRG}$ event sample are given in Table 5. The distributions have been corrected using a combination of Monte Carlo event samples generated by the POMPYT and the CDMBGF Monte Carlo generator. The relative normalisation of the Monte Carlo samples has been fixed by fitting the sum of the reconstructed $\eta_{\max }$ distribution from the POMPYT and the CDMBGF Monte Carlo sample to the measured $\eta_{\max }$ distribution [21].

Figure [b shows that the $x_{F}$ distribution becomes significantly softer with increasing $W$. The prediction of the QPM, where no scale breaking effects due to QCD radiation are included, almost agrees with the result from the fixed target experiments [7, 46, 47] but is very different from the result at HERA energies (dotted line in Fig. 6b). The effects of scaling violation in the $x_{F}$ distributions of hadrons, which have been found to be small when measured in a limited interval of $W$ and $Q^{2}\left[3\right.$, 5], become evident when studied over a large range of $W$ and $Q^{2}$. Models in which higher order $\alpha_{s}$ processes are considered (e.g. the MEPS model indicated by the full line in Fig. 6b) agree reasonably with the ZEUS data.

The mean value of $p_{t}^{* 2}$ as a function of $x_{F}$ is shown in Fig. 7 for $<W>=120 \mathrm{GeV}$ (this analysis) and for $\langle W\rangle=14 \mathrm{GeV}$ from the EMC collaboration 46 . Comparing the results at low $W$ and high $W$ there is a strong increase of $\left\langle p_{t}^{* 2}\right\rangle$ by a factor of about three over the whole range of $x_{F}>0.05$ going from $W=14$ to $120 \mathrm{GeV}$. The comparison of the prediction from the QPM and the models including higher order QCD processes shows that QCD effects are much larger at HERA energies than at energies reached in fixed target experiments.

For a further analysis of the $W$ and $Q^{2}$ dependence, $<p_{t}^{* 2}>$ was determined for two intervals in $x_{F}$ and four bins of $W$ at an average value for $Q^{2}$ of $28 \mathrm{GeV}^{2}$ (Fig. 8a) and four bins of $Q^{2}$ keeping $W$ fixed at an average value of $120 \mathrm{GeV}$ (Fig. 8 b). The value of $\left\langle p_{t}^{* 2}\right\rangle$ increases both with $W$ and with $Q^{2}$. The results are tabulated in the Tables 6 and 7 .

These results are compared with those from a fixed target experiment at lower energies [5, 48]. The rise of $\left\langle p_{t}^{* 2}>\right.$ with $W$, which had been observed already in the fixed target DIS experiments, continues in the range of $W$ seen at HERA. However, the $Q^{2}$-dependence in these two ranges of $W$ is different. There is a large overlap of the $Q^{2}$ intervals covered. At HERA energies a rise of $<p_{t}^{* 2}>$ with $Q^{2}$ is observed, while at low $W$ almost no dependence on $Q^{2}$ was found [5].

The results from the ZEUS experiment and the fixed target experiment are compared with model calculations in Fig. 9. The $W$-dependence is reasonably described by the MEPS (solid line) and CDMBGF models (dashed line). Also the colour dipole model without including the BGF process (dotted line) qualitatively reproduces the $W$ dependence of $<p_{t}^{* 2}>$ but overestimates the absolute value. The $Q^{2}$-dependence is also described by the MEPS and CDMBGF model but not by the colour dipole model (CDM) alone. The colour dipole model simulates higher order gluon radiation processes but the BGF process is not considered. The

$Q^{2}$ dependence of $\left\langle p_{t}^{* 2}>\right.$ shows that it is necessary to include the explicit treatment of the $\mathrm{BGF}$ process as well in the simulation.

\section{Conclusions}

Measurements of differential charged hadron multiplicity distributions in DIS events have been presented in the centre-of-mass system of the virtual photon and the proton at a centre-of-mass energy of $296 \mathrm{GeV}$ for $10 \leq Q^{2} \leq 160 \mathrm{GeV}^{2}$ and $75 \leq W \leq 175 \mathrm{GeV}$. 
The transverse momentum, $p_{t}^{*}$, and $x_{F}$ distributions have been investigated separately for events with (LRG) and without a large rapidity gap (NRG) between the proton direction and the observed hadronic final state. In the whole range of $x_{F}>0.05$ the values of $<p_{t}^{* 2}>$ for NRG events are much larger than those for the LRG events. These results confirm that gluon radiation in LRG events is strongly suppressed as compared to 'standard' DIS events at comparable $W$. A comparison of the data with the prediction of the QPM shows, however, that some QCD radiation is present also in LRG events.

The value of $\left\langle p_{t}^{* 2}>\right.$ in the LRG events is similar to that observed in deep inelastic $\mu p$ scattering experiments on fixed targets at low $W(<W>=14 \mathrm{GeV})$. This indicates that the multi-particle production in LRG events is similar to that in DIS at a scale of the final state invariant mass $W=M_{X}$, where $M_{X}$ is the invariant mass of the observed hadronic final state $X$, excluding the proton.

The comparison of the $x_{F}$ distributions in $e^{+} e^{-}$annihilation and in DIS events confirms the hypothesis that the hadron formation process in the current jet region is approximately independent of the type of the primary interacting particles.

The comparison of results presented here with those of DIS at low $W$ from fixed target experiments allows a study of the development of QCD effects in the $x_{F}$ and $p_{t}^{*}$ distributions over a large range in $W$ and $Q^{2}$. A significant increase of $<p_{t}^{* 2}>$ with $W$ is found. At HERA energies, the mean value of $p_{t}^{* 2}$ also rises with increasing $Q^{2}$ at fixed $W$. This can be understood in terms of the increase of the momentum space allowing the formation of more multi-jet events.

\section{Acknowledgements}

The experiment was made possible by the inventiveness and the diligent efforts of the HERA machine group who continued to run HERA most efficiently during 1993.

The design, construction and installation of the ZEUS detector has been made possible by the ingenuity and dedicated effort of many people from inside DESY and from the home institutes, who are not listed as authors. Their contributions are acknowledged with great appreciation.

The strong support and encouragement of the DESY Directorate has been invaluable.

We also gratefully acknowledge the support of the DESY computing and network services.

\section{References}

[1] N. Schmitz, Int. J. Mod. Phys. A8 (1993) 2026.

[2] ZEUS Collab., M. Derrick et al., Phys. Lett. B306 (1993) 158.

[3] EMC Collab., J.J. Aubert et al., Phys. Lett. B114 (1982) 373.

[4] EMC Collab., M. Arneodo et al., Nucl. Phys. B258 (1985) 249;

EMC Collab., M. Arneodo et al., Phys. Lett. B165 (1985) 222;

EMC Collab., M. Arneodo et al., Z. Phys. C31 (1986) 1. 
[5] EMC Collab., J. Ashman et al., Z. Phys. C52 (1991) 361.

[6] EMC Collab., J.J. Aubert et al., Phys. Lett. B95 (1980) 306.

[7] EMC Collab., M. Arneodo et al., Z. Phys. C36 (1986) 527.

[8] EMC Collab., M. Arneodo et al., Phys. Lett. B149 (1984) 415.

[9] ZEUS Collab., M. Derrick et al., Z. Phys. C67 (1995) 93.

[10] ZEUS Collab., M. Derrick et al., Phys. Lett. B315 (1993) 481.

[11] ZEUS Collab., 'The ZEUS Detector', Status Report 1993, DESY.

[12] ZEUS Collab., M. Derrick et al., Phys. Lett. B293 (1992) 465.

[13] C. Alvisi et al., Nucl. Instrum. Methods A305 (1991) 30.

[14] N. Harnew et al., Nucl. Instrum. Methods A279 (1989) 290;

B. Foster et al., Nucl. Phys. B (Proc. Suppl.) 32 (1993) 181;

B. Foster et al., Nucl. Instrum. Methods A338 (1994) 254.

[15] M. Derrick et al., Nucl. Instrum. Methods A309 (1991) 77;

A. Andresen et al., ibid. A309 (1991) 101;

A. Caldwell et al., ibid. A321 (1993) 356;

A. Bernstein et al., ibid. A336 (1993) 23.

[16] J. Andruszkòw et al., DESY 92-066 (1992).

[17] W.H. Smith et al., Nucl. Instrum. Methods A355 (1995) 278.

[18] S. Bentvelsen, J. Engelen and P. Kooijman, Proc. of the Workshop on Physics at HERA, DESY (1991) 23.

[19] F. Jacquet and A. Blondel, Proc. of the Workshop on Study of an ep Facility for Europe, DESY 79/48 (1979) 391.

[20] ZEUS Collab., M. Derrick et al., Z. Phys. C65 (1995) 379.

[21] ZEUS Collab., M. Derrick et al., Phys. Lett. B338 (1994) 483.

[22] R.E. Kalman, J. Basic Eng. 82 (1960) 35;

R.E. Kalman and R.S. Bucy, ibid. 83 (1961) 95;

P. Billoir and S. Qian, Nucl. Instrum. Methods A294 (1990) 219;

P. Billoir and S. Qian, ibid. A295 (1990) 492.

[23] P. Billoir and S. Quian, Nucl. Instrum. Methods A311 (1992) 139.

[24] G. Ingelman, Proc. of the Workshop on Physics at HERA, DESY (1991) 1366;

M. Bengtsson, G. Ingelman and T. Sjöstrand, Nucl. Phys. B301 (1988) 554.

[25] L. Lönnblad, Proc. of the Workshop on Physics at HERA, DESY (1991) 1440;

L. Lönnblad, Comp. Phys. Comm. 71 (1992) 15. 
[26] B. Andersson et al., Phys. Rep. 97 (1983) 31.

[27] B. Andersson et al., Z. Phys. C3 (1980) 223;

B. Andersson et al., ibid. C9 (1983) 233.

[28] T. Sjöstrand, Comp. Phys. Comm. 39 (1986) 347;

T. Sjöstrand, ibid. 43 (1987) 367.

[29] A. Kwiatowski, H. Spiesberger and H.-J. Möhring, Proc. of the Workshop on Physics at HERA, DESY (1991) 1294;

K. Charchuła, G. Schuler and H. Spiesberger, CERN-TH 7133/94.

[30] A.D. Martin, R.G. Roberts and W.J. Stirling, Phys. Lett. B306 (1993) 145.

[31] ZEUS Collab., M. Derrick et al., Z. Phys. C65 (1995) 379.

[32] H1 Collab., T. Ahmed et al., Nucl. Phys. B439 (1995) 471.

[33] G. Ingelman, Proceedings of the Europhysics Conference on High Energy Physics in Marseille, France, July 1993.

[34] H.-U. Bengtsson and T. Sjöstrand, Comp. Phys. Comm. 46 (1987) 43;

T. Sjöstrand, CERN-TH 6488/92.

[35] N.N. Nikolaev and B.G. Zakharov, Z. Phys. C53 (1992) 331.

[36] A. Solano, Ph. D. Thesis, University of Torino 1993, unpublished.

[37] R. Brun et al., Geant3, CERN DD/EE/84-1 (1987).

[38] H. Beier, Diploma thesis, Univ. of Hamburg (1994), DESY Int. Report, F35D 94-07.

[39] H1 Collab., I. Abt et al., Z. Phys. C63 (1994) 377.

[40] ZEUS Collab., M. Derrick et al., Phys. Lett. B332 (1994) 228.

[41] A. Donnachie and P.V. Landshoff, Nucl. Phys. B244 (1984) 322;

A. Donnachie and P.V. Landshoff, ibid. B267 (1985) 690.

[42] G. Ingelman and P. Schlein, Phys. Lett. B152 (1985) 256.

[43] N.N. Nikolaev and B.G. Zakharov, Z. Phys. C53 (1992) 331.

[44] DELPHI Collab., P. Abreu et al., Phys. Lett. B311 (1993) 408.

[45] R.D. Field and R. Feynman, Nucl. Phys. B136 (1978) 1.

[46] EMC Collab., M. Arneodo et al., Z. Phys. C35 (1986) 417.

[47] E665 Collab., M.R. Adams et al., FNAL-Pub 93/245-E (1993);

E665 Collab., M.R. Adams et al., Phys. Lett. B272 (1991) 163.

[48] U. Ecker, Ph.D. thesis, Univ. of Wuppertal, WUB-DIS 91-1 (1991);

E665 Collab., M.R. Adams et al., Phys. Lett. B272 (1991) 163. 


\begin{tabular}{|c|c|ccc|}
\hline \multicolumn{5}{|c|}{$\mathrm{A}$} \\
\hline$x_{F}$ & $<x_{F}>$ & \multicolumn{3}{|c|}{$\frac{1}{N_{\text {evt }}} \frac{d N_{\text {had }}}{d x_{F}}$} \\
\hline $0.03-0.05$ & 0.04 & 64.96 & \pm 0.78 & \pm 10.53 \\
$0.05-0.10$ & 0.07 & 27.92 & \pm 0.34 & \pm 4.27 \\
$0.10-0.15$ & 0.12 & 12.89 & \pm 0.23 & \pm 1.97 \\
$0.15-0.22$ & 0.18 & 6.67 & \pm 0.13 & \pm 0.97 \\
$0.22-0.32$ & 0.27 & 2.86 & \pm 0.07 & \pm 0.45 \\
$0.32-0.45$ & 0.38 & 1.15 & \pm 0.04 & \pm 0.15 \\
$0.45-0.65$ & 0.52 & 0.36 & \pm 0.02 & \pm 0.08 \\
$0.65-0.90$ & 0.73 & 0.07 & \pm 0.006 & \pm 0.02 \\
\hline \multicolumn{5}{|c|}{$\mathrm{B}$} \\
\hline$p_{t}^{*} \mathrm{GeV}$ & $<p_{t}^{*}>\mathrm{GeV}$ & $\frac{1}{N_{\text {evt }}} \frac{d N_{h a d}}{d p_{t}^{*}}$ & $\mathrm{GeV}^{-1}$ \\
\hline $0.00-0.10$ & 0.07 & 1.05 & \pm 0.05 & \pm 0.18 \\
$0.10-0.20$ & 0.15 & 2.84 & \pm 0.08 & \pm 0.51 \\
$0.20-0.40$ & 0.30 & 3.89 & \pm 0.07 & \pm 0.60 \\
$0.40-0.60$ & 0.49 & 3.34 & \pm 0.06 & \pm 0.47 \\
$0.60-0.80$ & 0.69 & 2.24 & \pm 0.05 & \pm 0.33 \\
$0.80-1.20$ & 0.96 & 1.10 & \pm 0.02 & \pm 0.18 \\
$1.20-1.50$ & 1.33 & 0.47 & \pm 0.02 & \pm 0.07 \\
$1.50-2.00$ & 1.71 & 0.23 & \pm 0.01 & \pm 0.03 \\
$2.00-2.75$ & 2.30 & 0.08 & \pm 0.004 & \pm 0.01 \\
$2.75-3.50$ & 3.07 & 0.03 & \pm 0.002 & \pm 0.004 \\
$3.50-5.00$ & 4.04 & 0.01 & \pm 0.001 & \pm 0.001 \\
\hline \multicolumn{5}{|c|}{$\mathrm{C}$} \\
\hline$x_{F}$ & $<x_{F}>$ & \multicolumn{5}{|c|}{$<p_{t}^{* 2}>\mathrm{GeV}^{2}$} \\
\hline $0.05-0.10$ & 0.07 & 0.47 & \pm 0.01 & \pm 0.02 \\
$0.10-0.15$ & 0.12 & 0.63 & \pm 0.02 & \pm 0.04 \\
$0.15-0.22$ & 0.18 & 0.85 & \pm 0.04 & \pm 0.03 \\
$0.22-0.32$ & 0.27 & 1.19 & \pm 0.06 & \pm 0.06 \\
$0.32-0.45$ & 0.38 & 1.50 & \pm 0.09 & \pm 0.13 \\
$0.45-0.65$ & 0.52 & 2.30 & \pm 0.19 & \pm 0.33 \\
$0.65-0.90$ & 0.73 & 2.09 & \pm 0.33 & \pm 0.66 \\
\hline \multicolumn{5}{|c|}{} \\
\hline
\end{tabular}

Table 3: Differential multiplicities for charged hadrons as a function of A) $x_{F}$ and B) $p_{t}^{*}$ $\left(x_{F}>0.05\right)$ and $\left.\mathrm{C}\right)<p_{t}^{* 2}>$ as a function of $x_{F}$ for DIS events with $\eta_{\max }>1.5$ (NRG) in the range of $10<Q^{2}<160 \mathrm{GeV}^{2}$ and $75<W<175 \mathrm{GeV}$. Statistical and systematic errors are given separately. 


\begin{tabular}{|c|c|ccc|}
\hline \multicolumn{5}{|c|}{$\mathbf{A}$} \\
\hline$x_{F}$ & $<x_{F}>$ & \multicolumn{3}{|c|}{$\frac{1}{N_{e v t}} \frac{d N_{\text {had }}}{d x_{F}}$} \\
\hline $0.03-0.05$ & 0.04 & 26.97 & \pm 1.88 & \pm 2.55 \\
$0.05-0.10$ & 0.07 & 19.70 & \pm 1.24 & \pm 0.94 \\
$0.10-0.15$ & 0.12 & 12.16 & \pm 1.02 & \pm 1.51 \\
$0.15-0.22$ & 0.18 & 6.49 & \pm 0.59 & \pm 1.01 \\
$0.22-0.32$ & 0.27 & 4.02 & \pm 0.45 & \pm 0.82 \\
$0.32-0.45$ & 0.38 & 1.41 & \pm 0.20 & \pm 0.46 \\
$0.45-0.65$ & 0.54 & 0.68 & \pm 0.12 & \pm 0.10 \\
$0.65-0.90$ & 0.75 & 0.31 & \pm 0.07 & \pm 0.07 \\
\hline \multicolumn{5}{|c|}{$\mathbf{B}$} \\
\hline$p_{t}^{*} \mathrm{GeV}$ & $<p_{t}^{*}>\mathrm{GeV}$ & $\frac{1}{N_{e v t}} \frac{d N_{h a d}}{d p_{t}^{*}}$ & $\mathrm{GeV}^{-1}$ \\
\hline $0.00-0.10$ & 0.07 & 1.36 & \pm 0.05 & \pm 0.50 \\
$0.10-0.20$ & 0.15 & 4.31 & \pm 0.11 & \pm 1.11 \\
$0.20-0.40$ & 0.30 & 4.91 & \pm 0.05 & \pm 0.41 \\
$0.40-0.60$ & 0.49 & 3.44 & \pm 0.04 & \pm 0.34 \\
$0.60-0.80$ & 0.68 & 1.45 & \pm 0.02 & \pm 0.55 \\
$0.80-1.20$ & 0.93 & 0.64 & \pm 0.01 & \pm 0.045 \\
$1.20-2.00$ & 1.41 & 0.09 & \pm 0.003 & \pm 0.02 \\
$2.00-5.00$ & 3.86 & 0.001 & \pm 0.001 & \pm 0.004 \\
\hline \multicolumn{5}{|c|}{$\mathrm{C}$} \\
\hline$x_{F}$ & $<x_{F}>$ & \multicolumn{3}{|c|}{$<p_{t}^{* 2}>\mathrm{GeV}^{2}$} \\
\hline $0.05-0.10$ & 0.07 & 0.19 & \pm 0.02 & \pm 0.01 \\
$0.10-0.15$ & 0.12 & 0.24 & \pm 0.05 & \pm 0.03 \\
$0.15-0.22$ & 0.18 & 0.35 & \pm 0.08 & \pm 0.04 \\
$0.22-0.32$ & 0.27 & 0.41 & \pm 0.11 & \pm 0.16 \\
$0.32-0.45$ & 0.38 & 0.38 & \pm 0.07 & \pm 0.11 \\
$0.45-0.65$ & 0.54 & 0.50 & \pm 0.10 & \pm 0.12 \\
$0.65-0.90$ & 0.75 & 0.37 & \pm 0.13 & \pm 0.60 \\
\hline
\end{tabular}

Table 4: Differential multiplicities for charged hadrons as a function of A) $x_{F}$ and B) $p_{t}^{*}$ $\left(x_{F}>0.05\right)$ and $\left.\mathrm{C}\right)<p_{t}^{* 2}>$ as a function of $x_{F}$ for DIS events with $\eta_{\max }<1.5$ (LRG) in the range of $10<Q^{2}<160 \mathrm{GeV}^{2}$ and $75<W<175 \mathrm{GeV}$. Statistical and systematic errors are given separately. 


\begin{tabular}{|c|c|ccc|}
\hline \multicolumn{5}{|c|}{$\mathrm{A}$} \\
\hline$x_{F}$ & $<x_{F}>$ & \multicolumn{3}{c|}{$\frac{1}{N_{\text {evt }}} \frac{d N_{\text {had }}}{d x_{F}}$} \\
\hline $0.03-0.05$ & 0.04 & 62.99 & \pm 0.75 & \pm 9.59 \\
$0.05-0.10$ & 0.07 & 27.48 & \pm 0.33 & \pm 4.03 \\
$0.10-0.15$ & 0.12 & 12.86 & \pm 0.22 & \pm 1.99 \\
$0.15-0.22$ & 0.18 & 6.67 & \pm 0.13 & \pm 0.98 \\
$0.22-0.32$ & 0.27 & 2.90 & \pm 0.07 & \pm 0.46 \\
$0.32-0.45$ & 0.38 & 1.18 & \pm 0.04 & \pm 0.17 \\
$0.45-0.65$ & 0.52 & 0.37 & \pm 0.02 & \pm 0.08 \\
$0.65-0.90$ & 0.73 & 0.08 & \pm 0.007 & \pm 0.03 \\
\hline \multicolumn{5}{|c|}{$\mathrm{B}$} \\
\hline$p_{t}^{*} \mathrm{GeV}$ & $<p_{t}^{*}>\mathrm{GeV}$ & $\frac{1}{N_{\text {evt }}} \frac{d N_{h a d}}{d p_{t}^{*}}$ & $\mathrm{GeV}^{-1}$ \\
\hline $0.00-0.10$ & 0.07 & 1.10 & \pm 0.05 & \pm 0.18 \\
$0.10-0.20$ & 0.15 & 2.92 & \pm 0.08 & \pm 0.52 \\
$0.20-0.40$ & 0.30 & 3.98 & \pm 0.07 & \pm 0.60 \\
$0.40-0.60$ & 0.49 & 3.35 & \pm 0.06 & \pm 0.47 \\
$0.60-0.80$ & 0.69 & 2.22 & \pm 0.05 & \pm 0.32 \\
$0.80-1.20$ & 0.96 & 1.07 & \pm 0.02 & \pm 0.18 \\
$1.20-1.50$ & 1.33 & 0.45 & \pm 0.02 & \pm 0.08 \\
$1.50-2.00$ & 1.71 & 0.22 & \pm 0.01 & \pm 0.03 \\
$2.00-2.75$ & 2.30 & 0.07 & \pm 0.004 & \pm 0.01 \\
$2.75-3.50$ & 3.07 & 0.02 & \pm 0.002 & \pm 0.004 \\
$3.50-5.00$ & 4.04 & 0.01 & \pm 0.001 & \pm 0.001 \\
\hline \multicolumn{5}{|c|}{$\mathrm{C}$} \\
\hline$x_{F}$ & $<x_{F}>$ & \multicolumn{5}{|c|}{$<p_{t}^{* 2}>\mathrm{GeV}^{2}$} \\
\hline $0.05-0.10$ & 0.07 & 0.46 & \pm 0.01 & \pm 0.02 \\
$0.10-0.15$ & 0.12 & 0.61 & \pm 0.02 & \pm 0.04 \\
$0.15-0.22$ & 0.18 & 0.82 & \pm 0.04 & \pm 0.03 \\
$0.22-0.32$ & 0.27 & 1.14 & \pm 0.06 & \pm 0.06 \\
$0.32-0.45$ & 0.38 & 1.40 & \pm 0.08 & \pm 0.11 \\
$0.45-0.65$ & 0.52 & 2.13 & \pm 0.18 & \pm 0.39 \\
$0.65-0.90$ & 0.73 & 1.82 & \pm 0.28 & \pm 0.56 \\
\hline \multicolumn{5}{|c|}{} \\
\hline
\end{tabular}

Table 5: Differential multiplicities for charged hadrons as a function of A) $x_{F}$ and B) $p_{t}^{*}$ $\left(x_{F}>0.05\right)$ and $\left.\mathrm{C}\right)<p_{t}^{* 2}>$ as a function of $x_{F}$ for DIS events (combined NRG + LRG event sample) in the range of $10<Q^{2}<160 \mathrm{GeV}^{2}$ and $75<W<175 \mathrm{GeV}$. Statistical and systematic errors are given separately. 


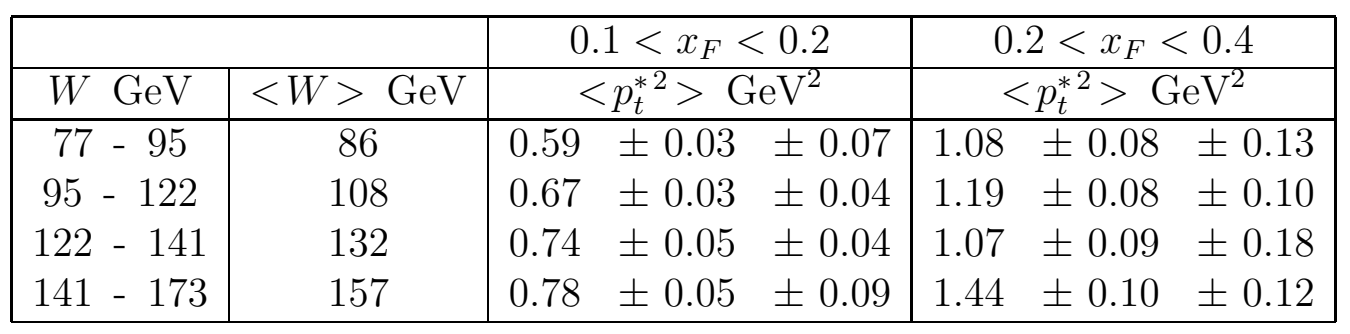

Table 6: $\left\langle p_{t}^{* 2}\right\rangle$ as a function of $W$ in two intervals of $x_{F}$. Statistical and systematic errors are given separately.

\begin{tabular}{|c|c|c|c|c|c|c|c|}
\hline & \multirow{2}{*}{\multicolumn{3}{|c|}{$\begin{array}{l}0.1<x_{F}<0.2 \\
<p_{t}^{* 2}>\mathrm{GeV}^{2}\end{array}$}} & \multirow{2}{*}{\multicolumn{3}{|c|}{$\begin{array}{l}0.2<x_{F}<0.4 \\
<p_{t}^{* 2}>\mathrm{GeV}^{2}\end{array}$}} \\
\hline$Q^{2} \mathrm{GeV}^{2}$ & $\left\langle Q^{2}>\mathrm{GeV}\right.$ & & & & & & \\
\hline $10-20$ & 14 & 0.59 & \pm 0.02 & \pm 0.03 & 1.05 & \pm 0.0 & \pm 0.07 \\
\hline $20-40$ & 28 & 77 & \pm 0.0 & \pm & 1.2 & \pm & 04 \\
\hline $40-80$ & 54 & 0.86 & \pm 0.08 & \pm 0 & 1.46 & \pm 0 & \pm 0.17 \\
\hline 80 & 110 & 0.96 & \pm 0.11 & \pm 0.15 & 2.12 & \pm 0.3 & \pm 0.77 \\
\hline
\end{tabular}

Table 7: $\quad\left\langle p_{t}^{* 2}>\right.$ as a function of $Q^{2}$ in two intervals of $x_{F}$. Statistical and systematic errors are given separately. 


\section{ZEUS 1993}

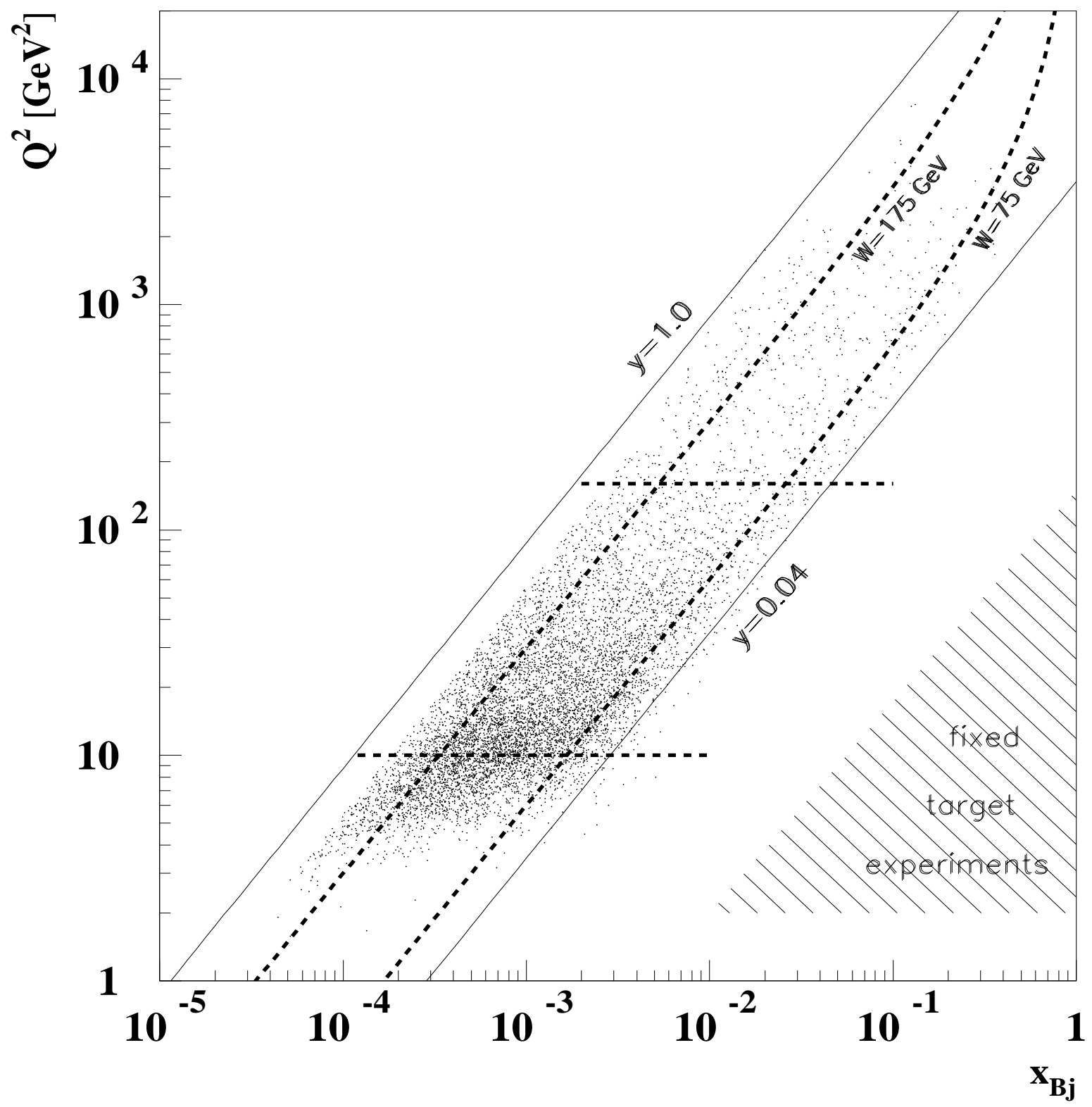

Figure 1: Population of the $Q^{2}-x$ plane by the DIS events selected for this analysis. For the sake of clarity only $1 / 3$ of the DIS event sample is shown in the scatter plot. Charged hadron distributions are investigated for $10<Q^{2}<160 \mathrm{GeV}^{2}$ and $75<W<175 \mathrm{GeV}$ (dashed lines). The approximate kinematic region covered by the fixed target experiments is also indicated. 


\section{ZEUS 1993}

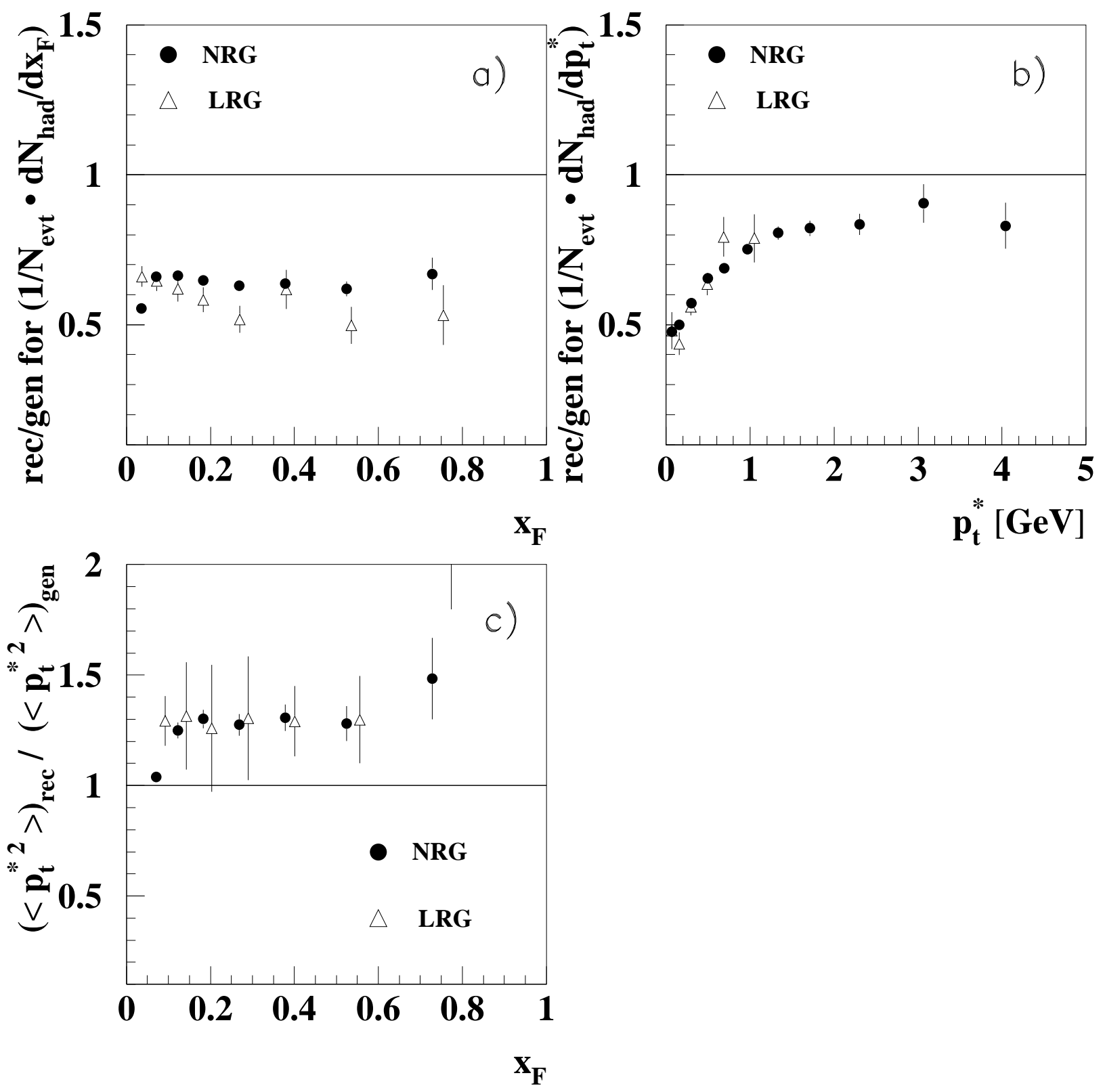

Figure 2: Inverse of the correction functions $c(v)$ for a) the $x_{F}$ and $\mathrm{b}$ ) the $p_{t}^{*}$ distribution in the range of $10<Q^{2}<160 \mathrm{GeV}^{2}$ and $75<W<175 \mathrm{GeV}$, which are used to correct the NRG event sample (full points) and the LRG event sample (triangles). The inverse of the correction function for $<p_{t}^{* 2}>$ as a function of $x_{F}$ in the same range of $Q^{2}$ and $W$ is shown in c). 


\section{ZEUS 1993}
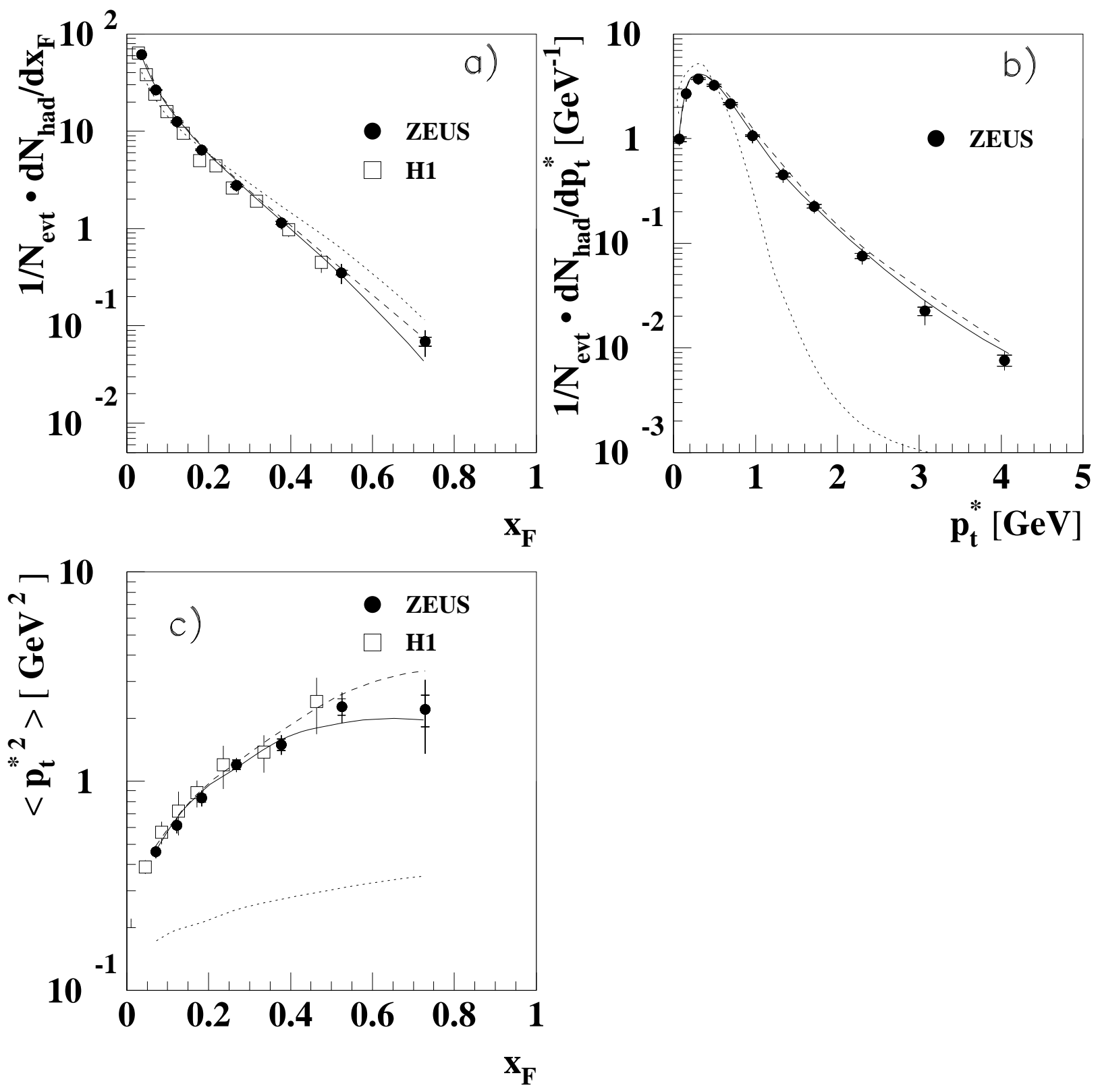

Figure 3: Differential charged hadron multiplicities for NRG DIS events normalised by the number of events as a function of a) $x_{F}$ and b) $p_{t}^{*}$ for $x_{F}>0.05$. c) $<p_{t}^{* 2}>$ as a function of $x_{F}$. For all plots the events are in a range $10 \leq Q^{2} \leq 160 \mathrm{GeV}^{2}$ and $75 \leq W \leq 175 \mathrm{GeV}$. The predictions of two DIS Monte Carlo models including QCD processes are shown: the MEPS model (solid curve) and the CDMBGF model (dashed curve). The prediction of the QPM is given by the dotted curve. The results of this analysis in a) and c) are also compared to measurements of the $\mathrm{H} 1$ collaboration 39 . 


\section{ZEUS 1993}
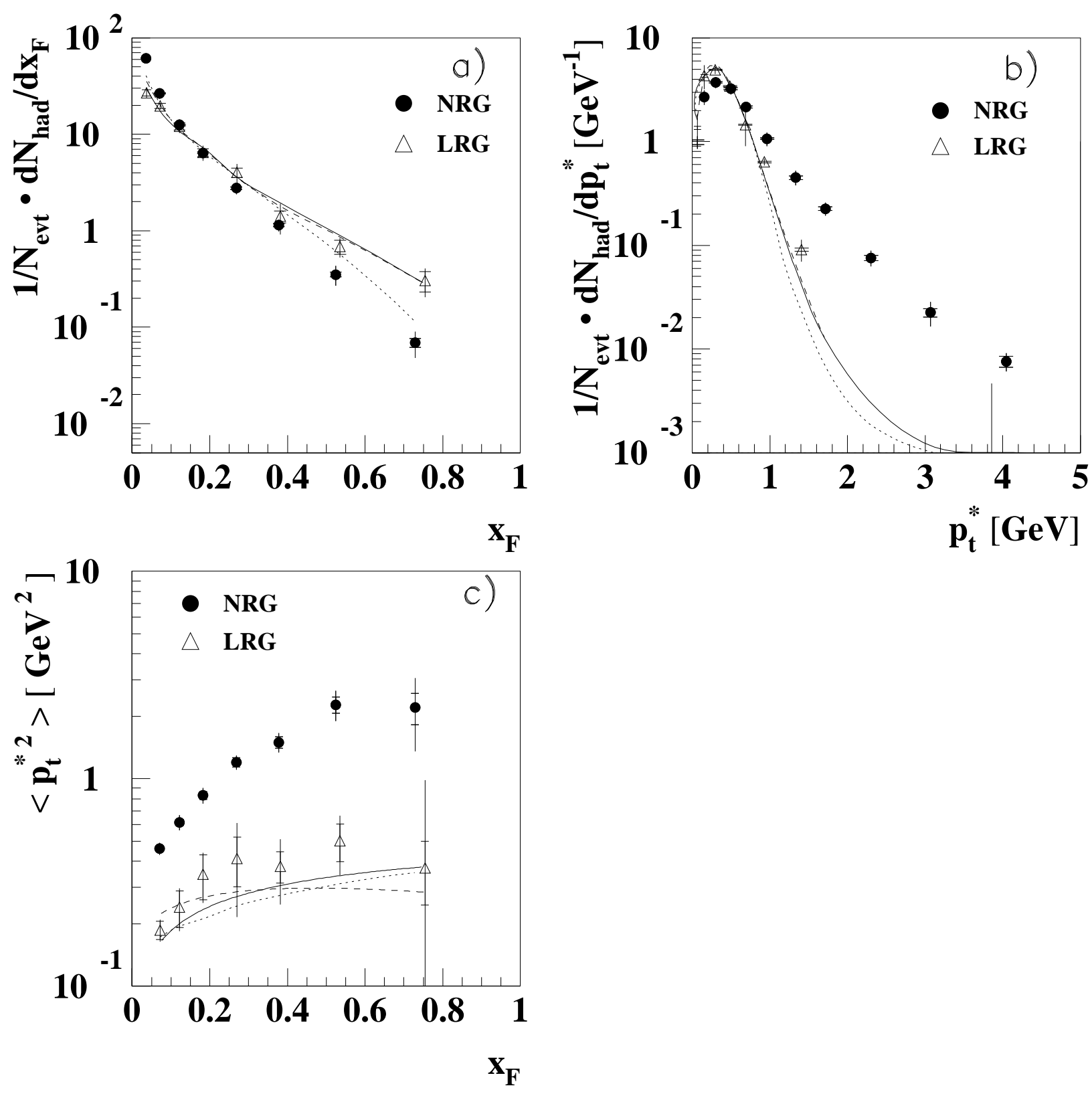

Figure 4: Charged hardon distributions for $10 \leq Q^{2} \leq 160 \mathrm{GeV}^{2}$ and $75 \leq W \leq 175 \mathrm{GeV}$ $\left(<Q^{2}>=28 \mathrm{GeV}^{2}\right.$ and $\left.\langle W\rangle=120 \mathrm{GeV}\right)$. a) The $x_{F}$ distribution, b) the $p_{t}^{*}$ distribution for $x_{F}>0.05$ and $\left.\mathrm{c}\right)<p_{t}^{* 2}>$ as a function of $x_{F}$ are presented separately for NRG and LRG events. In all three figures the curves represent the results of the following model predictions: solid curve: POMPYT with a hard pomeron structure function (see Table Q); dashed curve: model of Nikolaev and Zakharov; dotted curve: QPM. 


\section{ZEUS 1993}
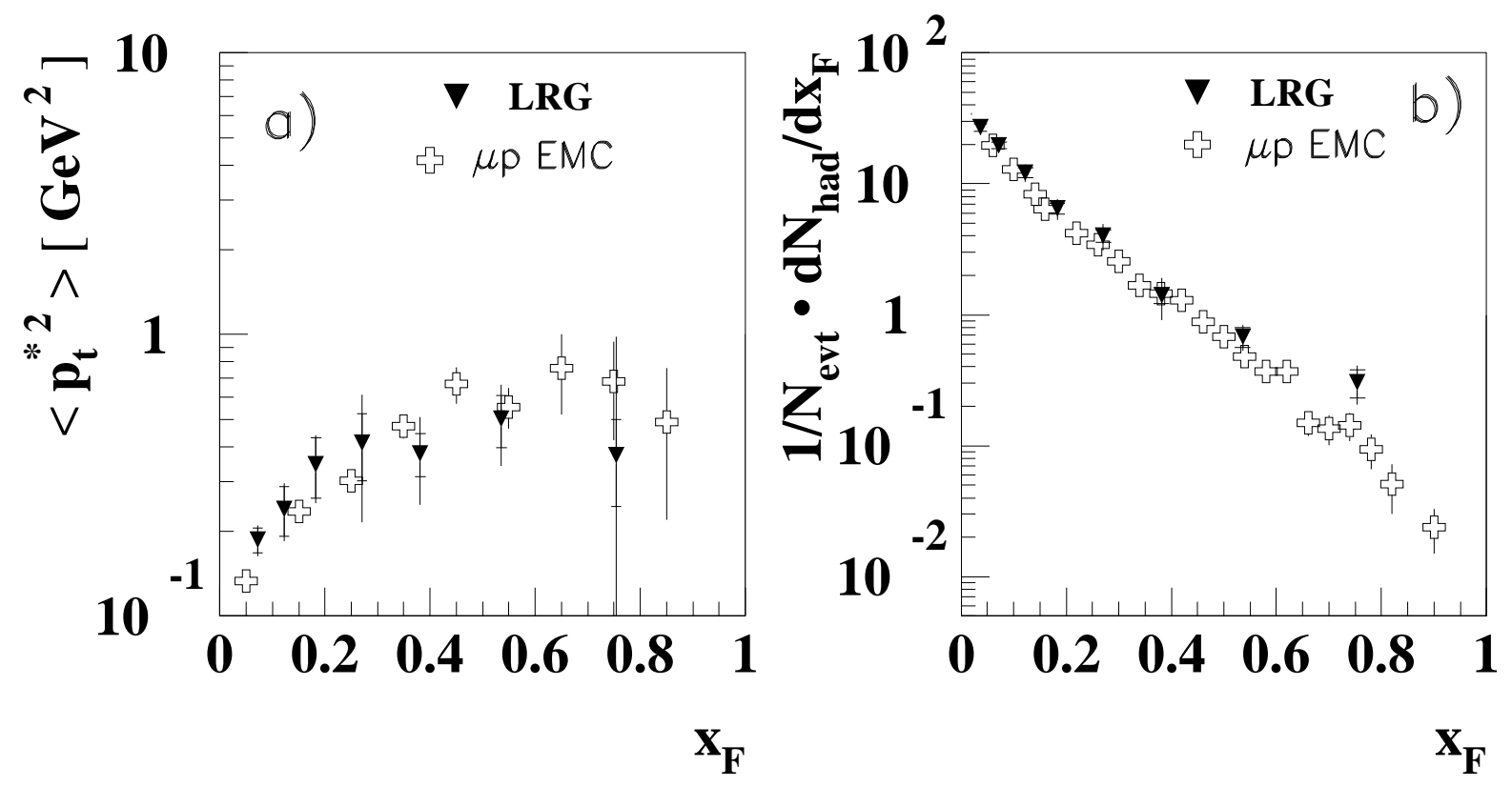

Figure 5: Comparison of a) $<p_{t}^{* 2}>$ as a function of $x_{F}$ and b) the $x_{F}$ distribution for the LRG event sample (ZEUS) and DIS at low energy (EMC, $<W>=14 \mathrm{GeV}$ ). The mean value of $M_{X}$ for the LRG event sample is $\left\langle M_{X}\right\rangle=8 \mathrm{GeV}$. 


\section{ZEUS 1993}
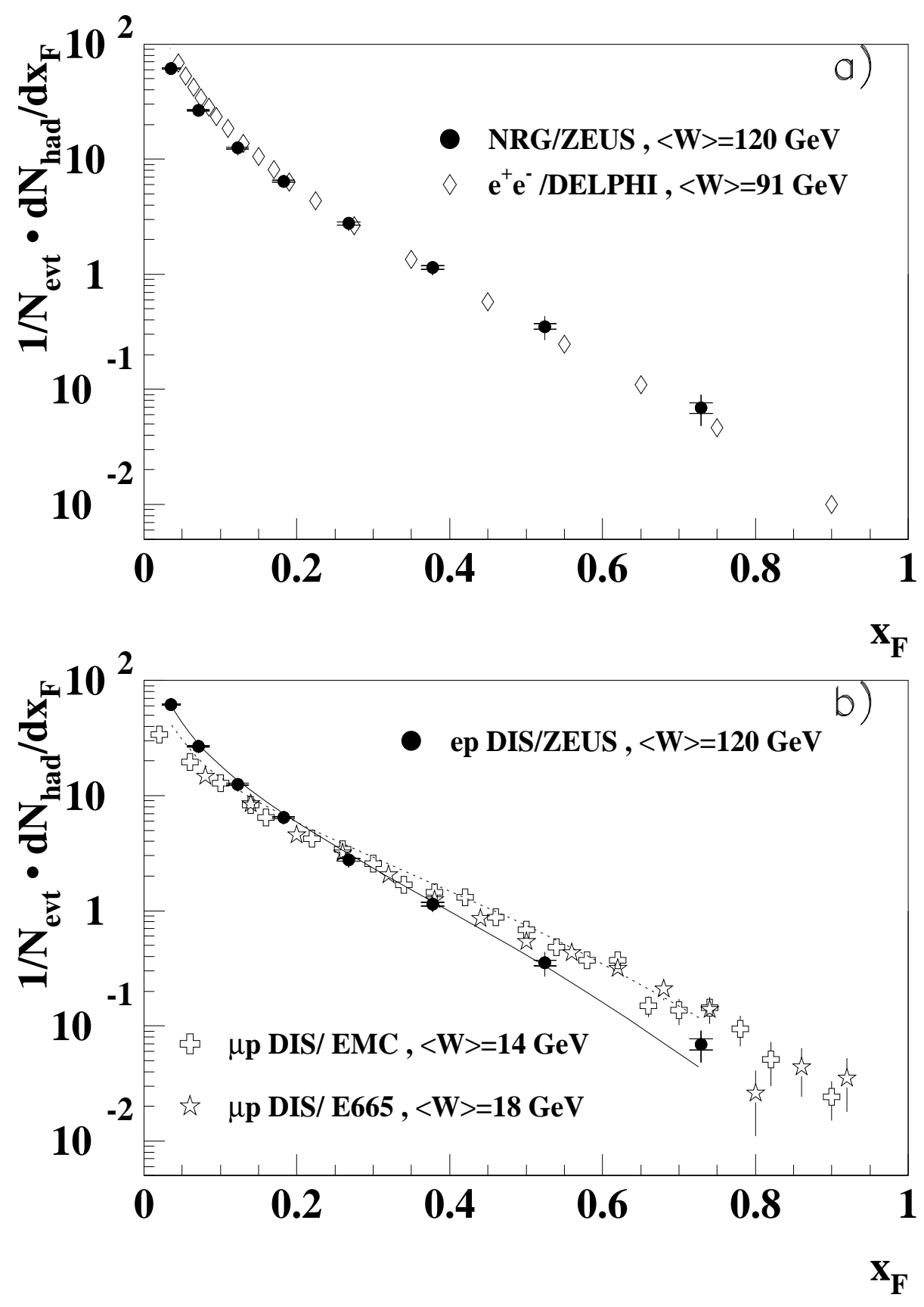

Figure 6: a) $x_{F}$ distribution from this analysis (NRG events) compared to results from $e^{+} e^{-}$ annihilation on the $Z^{0}$ resonance $(W=91 \mathrm{GeV})$ [4]. b) $x_{F}$ distribution from this analysis (NRG + LRG events) compared with results from $\mu p$ DIS at $<W>=14 \mathrm{GeV}$ 46] and at $<W>=18 \mathrm{GeV}$ [47]. In Fig. 6b) the solid curve shows the prediction of the MEPS model calculation and the dotted curve that of the QPM at HERA energies. 


\section{ZEUS 1993}

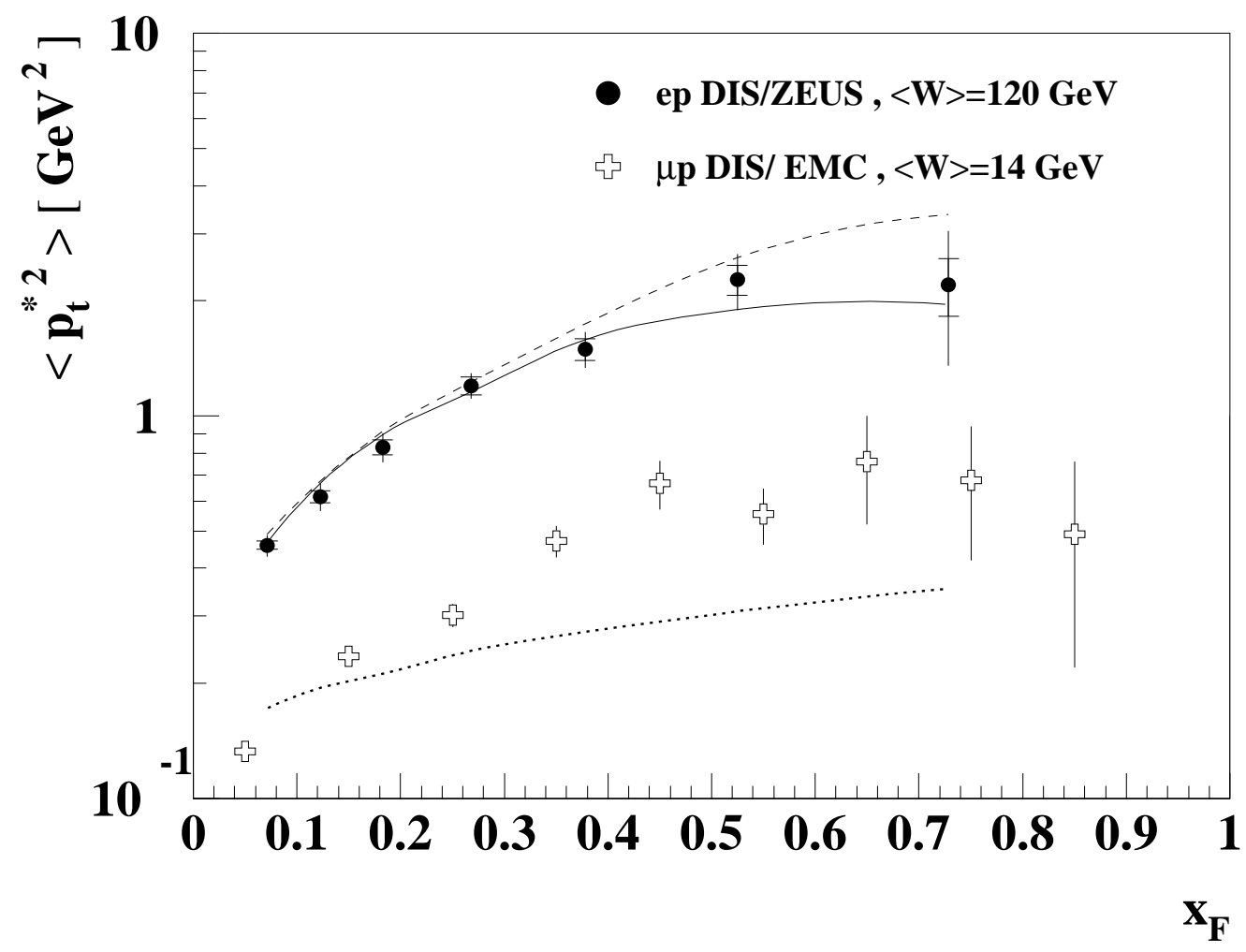

Figure 7: $\left\langle p_{t}^{* 2}>\right.$ as a function of $x_{F}$ from this analysis (NRG + LRG events) compared to results from $\mu p$ DIS at $\langle W\rangle=14 \mathrm{GeV}$ [46]. The curves show results from model calculations at HERA energy with the MEPS model (solid curve), the CDMBGF model (dashed curve) and the QPM (dotted curve). 


\section{ZEUS 1993}
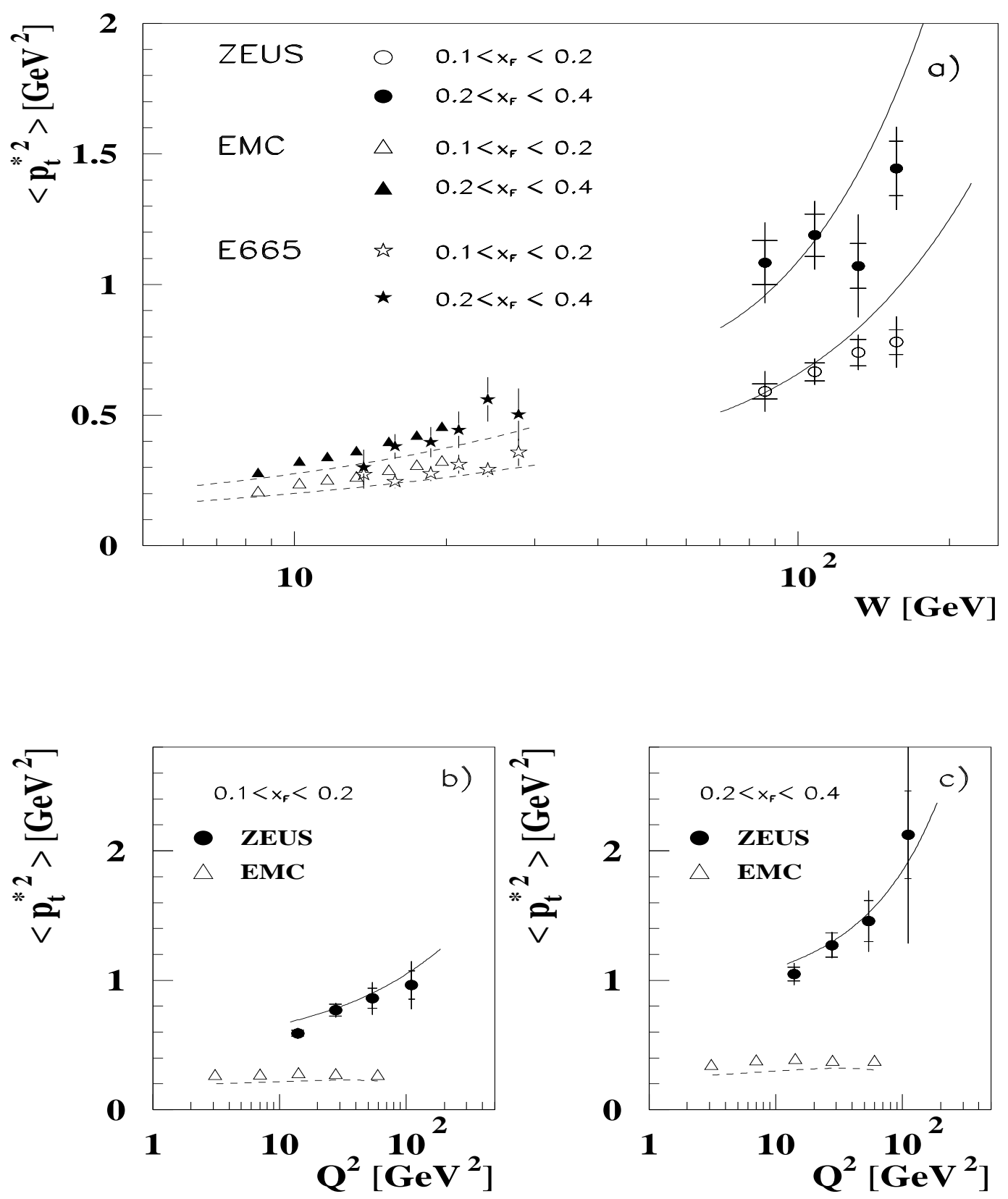

Figure 8: $\quad\left\langle p_{t}^{* 2}>\right.$ in two intervals of $x_{F}$ as a function of a) $W$ and b),c) $Q^{2}$ compared with results from $\mu p$ DIS experiments (EMC [5] and E665 [48]). The prediction of the MEPS Monte Carlo calculation is compared with the results of this analysis (solid curve) and of [5] (dashed curve). 


\section{ZEUS 1993}
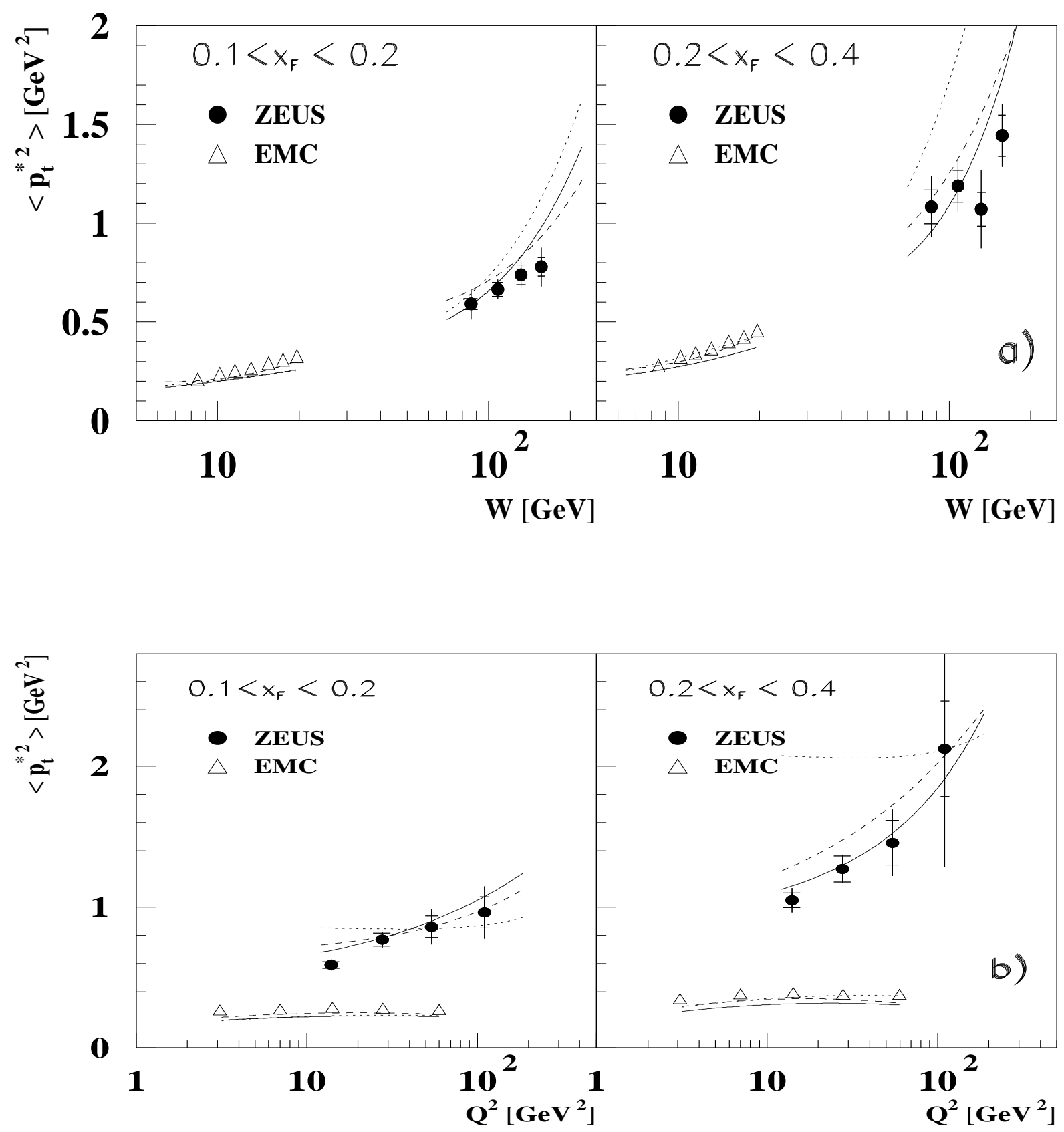

Figure 9: a) $W$ and b) $Q^{2}$ dependence of $<p_{t}^{* 2}>$ from EMC [5] and ZEUS data compared with different model predictions for the hadron formation: MEPS (solid curve), CDMBGF (dashed curve) and CDM (dotted curve). For the explanation of acronyms see Table 2. 